\title{
THREE-DIMENSIONAL COSSERAT HOMOGENIZATION OF MASONRY STRUCTURES: Elasticity
}

I. Stefanou ${ }^{1}$, J. Sulem ${ }^{2}$ and I. Vardoulakis ${ }^{3}$

${ }^{1}$ Department of Applied Mechanics and Physics, National Technical University of Athens, Greece, istefanou@mechan.ntua.gr

${ }^{2}$ CERMES, Ecole Nationale des Ponts et Chaussées/LCPC, Institut Navier, Paris, France, sulem@cermes.enpc.fr

${ }^{3}$ Department of Applied Mechanics and Physics, National Technical University of Athens, Greece, I.Vardoulakis@mechan.ntua.gr

Abstract: Masonry is a two-phase composite material formed by regularly distributed bricks and mortar. The homogenization procedure followed here extends the 2D approach of Sulem and Mühlhaus [4] and leads to an anisotropic 3D Cosserat continuum. The enriched kinematics of the Cosserat continuum allow to model microelement systems undergoing in-plane and out-of-plane rotations. The domain of validity of the derived Cosserat continuum is discussed by comparing the dispersion function of the discrete system of blocks with the continuous one and is found to be in good agreement.

Key words: masonry, 3D Cosserat, out of plane, in plane, homogenization, differential expansions.

\section{Introduction}

Masonry as a whole is more than the sum of its building blocks. Masonry is an anisotropic, composite material of bricks and mortar, which ideally can be seen as a periodic medium. Its mechanical behavior has been studied experimentally and analytically. Many models have been proposed for the description of the mechanical behavior of masonry walls based either on experimental results and empirical statements or on sophisticated continuum models that consider the micro-structure of the medium (see Besdo [1], Masiani et al. [2], Masiani \& Trovalusci [3], Sulem \& Mühlhaus [4], Pradel \& Sab [5], Cecchi \& Sab [6] for 2D masonry and Cecchi \& Sab [7]-[8], Trovalusci \& Masiani [9] for 3D masonry). 
The latter continuum models are derived by homogenization of the micro-structure.

The homogenization techniques are based on different methods for the representation of a periodically heterogeneous medium with a macroscopically equivalent homogeneous one. The most popular methods for homogenizing a medium are the following:

a) Asymptotic averaging methods. These methods are based on the asymptotic expansion of the state fields (displacements, forces, moments, etc.) in terms of a small quantity $\varepsilon$, which is the characteristic size of the elementary volume. The main advantage of these methods is their rigorous mathematical formulation and their ability to determine the error of the approximation of the discrete medium by the homogenized one. These methods are very popular for periodic composite materials and many applications can be found at Bensoussan et al.[10], Sanchez-Palencia [11], Sanchez-Palencia \& Zaoui [12], Bakhalov \& Panasenko [13], Kalamkarov [14], Tollenaere \& Caillerie [15].

b) Direct averaging methods. These methods are based on the direct averaging of strains and stresses over the representative volume element. Historically, these methods have been developed earlier than the asymptotic averaging methods and are discussed in details in Hashin [16], Hashin \& Shtrikman [17], Hill [18]-[19], Christensen [20], Aboudi [21].

c) Homogenization by integral transformations. This method was originally proposed by Kunin [22], [23] and is based on the substitution of the periodic heterogeneous medium with a continuous one, whose field variables coincide with the discrete ones at its nodes. Between the nodes, the values are given by trigonometrical interpolation.

d) Homogenization by differential expansions. This method [4], [24]-[28] is based on the derivation of a continuous model by replacing the difference quotients of the equations that describe the periodic heterogeneous medium with corresponding differential ones. This replacement is performed using Taylor expansions of desired order. According to Pasternak \& Mühlhaus [29], this 
method offers a robust balance between accuracy and simplicity and it is a longwave asymptotic approximation of the discrete, exact model.

The latter of the aforementioned, briefly described homogenization techniques, has been successfully applied in rock mechanics, in soil mechanics and in structural engineering. More specifically, continuum models have been derived in rock mechanics for the modeling of blocky rock masses by Mühlhaus [30]; in soil mechanics for the modeling of granular materials by Mühlhaus \& Oka [24] and Suiker et al. [26]-[28]; and in structural engineering for the two-dimensional modeling of classical ancient columns and masonry walls, Mühlhaus et al. [31], Sulem \& Mühlhaus [4], Cerrolaza et al. [32].

Here, we extend the continuum model for masonry walls in three-dimensions [33]. The interest of 3D extension is not only to describe in-plane deformations but also to account for out-of-plane deformation modes. Out-of plane deformation has often been observed to be of great importance for ancient masonry walls, which, among others, are the Parthenon structural Wall and the Acropolis retaining Wall. This study is devoted to the derivation and the validation of the model, whereas in a future work the derived constitutive law will be introduced into a finite element code in order to analyse real and complex structures.

It should be mentioned that the applications of the derived model and of the method presented here are not limited to masonry structures only. Applications may also be found to some geo-structures encountered in geotechnical and rock engineering, i.e. retaining walls, tunneling in blocky rocks, rock slopes (cf. e.g. Adhikary [34]) etc.. An additional example where this theory might be of interest is the modelling of periodic structures in natural limestone deposits that resemble to masonry structures and are of interest to structural geology. Generally, one could maintain that this theory might be useful in the modelling of soils that exhibit microstructures that can be represented as brick assemblages.

In section 1 we start from the micro-scale of the masonry wall and formulate the lattice model of the structure. In the next section, the discrete medium is homogenized using the differential expansions technique and the elastic potentials of the models. The constitutive law of the continuous model is then derived. In 
section 3 the derived Cosserat continuum is compared to the lattice one, in terms of the dispersion function, and it is found to approximate well the lattice model. All the analytical calculations in the present paper have been performed ${ }^{1}$ with the symbolic language mathematical package MATHEMATICA 5 [36].

\section{The lattice model}

Lattice models originally appeared in the context of condensed matter physics, where the atoms of a crystal directly form a lattice. Lattice models are quite popular in physics and mechanics as they have inspired many applications and approaches. The well-known Discrete Element Method, which is derived from molecular dynamics algorithms, could also be seen as an extended lattice model. Generally lattice models are ideal in computational physics and mechanics, as the discretisation of any continuum model automatically turns into a lattice model. A masonry wall can be regarded as a set of blocks which are regularly and periodically arranged in space. This regular periodic arrangement of the building blocks is suitable for the description of the brickwork by lattice models.

Starting from the micro-scale, i.e. the arrangement of the building blocks, the proposed model describes the macroscopic behavior of the wall by assuming rigid building blocks with deformable interfaces (soft-contacts). It should be mentioned that this hypothesis implies that the deformation is concentrated on the interfaces of the bricks and that it is small as compared to their dimensions (small strains assumption), which is verified especially in historical masonry structures. Raffard [37] has experimentally shown that the rigidity of the interface (brick-mortarbrick) is smaller than the rigidity of the mortar itself. According to Raffard this may be attributed to an increased porosity at the interface mortar-brick. The assumption of rigid building blocks with deformable interfaces is also adopted by many other researchers in similar considerations (Besdo [1], Masiani et al. [2], Masiani \& Trovalusci [3], Sulem \& Mühlhaus [4], Cecchi \& Sab [6]-[8],

1 The reader is invited to download the Mathematica Working files from: http://geolab.mechan.ntua.gr/people/stefanou. 
Trovalusci \& Masiani [9] just to mention some). On the other hand, the consideration of deformable blocks in the homogenization procedure would result in a higher order equivalent continuum with deformable directors. The scope of the paper is, however, to restrict the obtained homogenized medium to a Cosserat continuum. An additional assumption is that the bed and the cross joints of the brickwork (horizontal and vertical interfaces accordingly) have the same mechanical properties. Finally, the developed stresses $\Sigma^{b}$ at the interfaces of the blocks are assumed to be linearly distributed over them, and the constitutive law of the joints is assumed to be linear elastic (Figure 1). The assumption of linear stress distribution is justified in the recent publication of Milani et al. [38], where the authors show that linear stress distributions at the interfaces give good results as compared to constant and quadratic stress distributions.
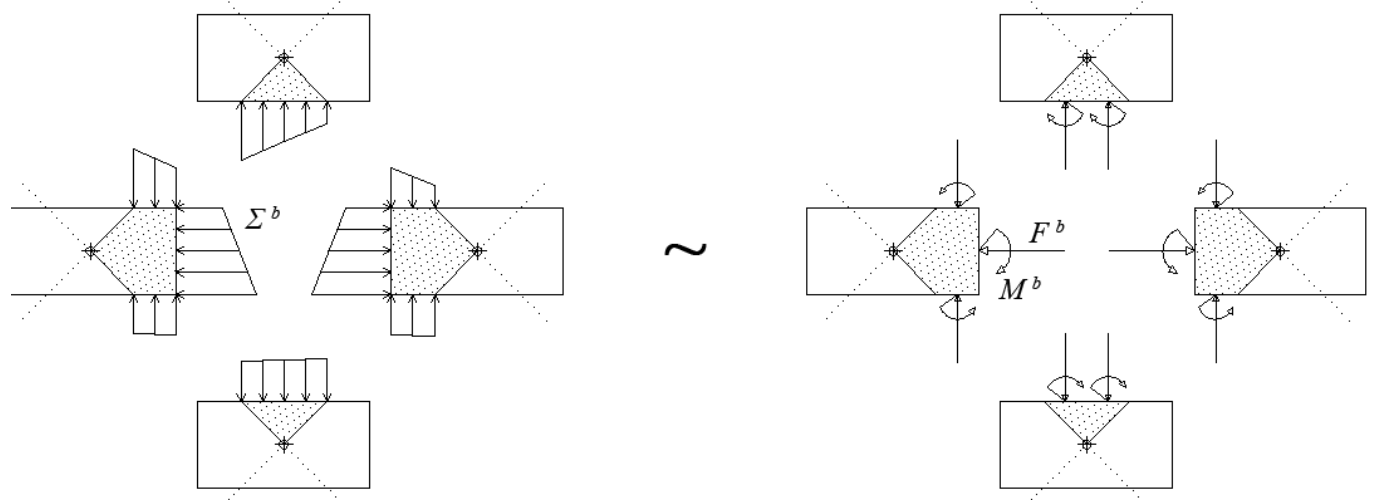

Figure 1. Stresses developed at the interfaces of the blocks of the elementary cell and their equivalent forces and moments.

The six degrees of freedom of each rigid building block can be separated into two groups describing the in plane and the out of plane deformation of the masonry wall. Accordingly, the nodes of the lattice model, which are fixed at the center of the masses of the building blocks, have six degrees of freedom. Obviously, the arrangement of the nodes of the lattice model is periodic in space (Figure 2) and follows a given pattern. We call this pattern the "elementary cell" and we define it as the minimum recurrent volume of the structure that contains all the necessary information for the constitutive description of the material. 
It has to be mentioned though, that generally the elementary cell is not unique and that its choice affects the obtained homogenized continuum. For this rather well-known point we refer to the book of Novozhilov 1961, [39]. In the particular case of masonry walls one could alternatively choose as an elementary cell an individual block with six interfaces and would indeed result in a constitutive law with small differences in the coefficients of the couple stresses. However, these differences are insignificant when these two approaches are compared with the discrete model in terms of the dispersion function.

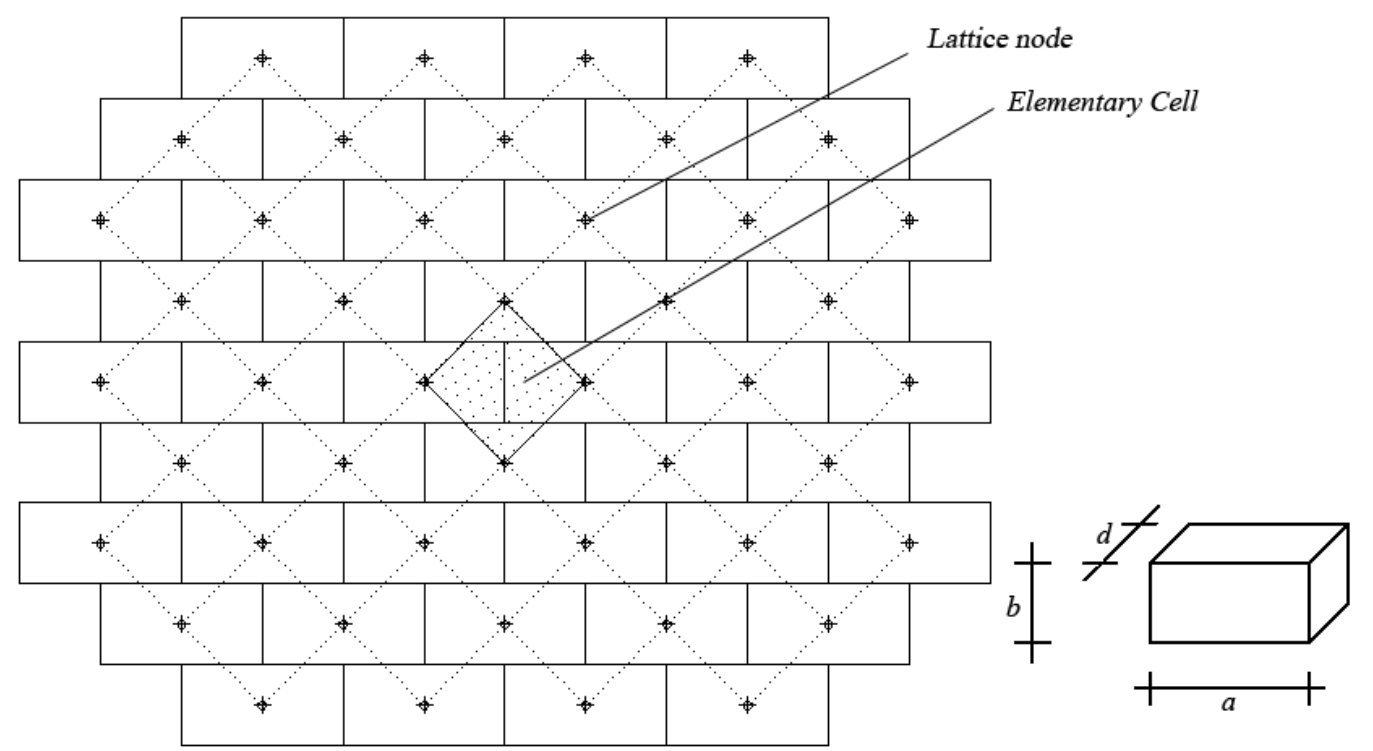

Figure 2. Chosen elementary cell for the periodic masonry structure.

\section{Internal forces}

Each block $(\mathrm{I}, \mathrm{J})$ of the lattice has three translational, $\mathbf{u}_{\mathrm{I}, \mathrm{J}}^{b}$, and three rotational, $\boldsymbol{\omega}_{\mathrm{I}, \mathrm{J}}^{b}$, degrees of freedom (Lagrange coordinates). $\mathbf{u}_{\mathrm{I}, \mathrm{J}}^{b}$ and $\boldsymbol{\omega}_{\mathrm{I}, \mathrm{J}}^{b}$ represent the displacement and the rotation of the center of mass of block (I,J). For infinitesimal rotations:

$$
\mathbf{u}_{\mathrm{I}, \mathrm{J}}^{b}(\mathbf{r})=\mathbf{u}_{\mathrm{I}, \mathrm{J}}^{b}+\boldsymbol{\omega}_{\mathrm{I}, \mathrm{J}}^{b} \times\left(\mathbf{r}-\mathbf{r}_{\mathrm{I}, \mathrm{J}}\right)
$$


where $\mathbf{u}_{\mathrm{I}, \mathrm{J}}^{b}(\mathbf{r})$ is the displacement vector of a point of block $(\mathrm{I}, \mathrm{J})$ with position vector $\mathbf{r}$ and $\mathbf{r}_{\mathrm{I}, \mathrm{J}}$ is the position vector of the center of mass of the block $(\mathrm{I}, \mathrm{J})$. Both $\mathbf{r}$ and $\mathbf{r}_{\mathrm{I}, \mathrm{J}}$ are expressed in the global coordinate system.

The elementary cell contains five interfaces $\Sigma_{k}(k=1,5)$, which are defined below in reference to the center of mass of the elementary cell (Figure 1, Figure 3):

$$
\begin{gathered}
\Sigma_{(1)}=\left(\begin{array}{c}
0 \leq x_{1} \leq \frac{a}{4} \\
x_{2}=\frac{b}{2} \\
-\frac{d}{2} \leq x_{3} \leq \frac{d}{2}
\end{array}\right), \quad \Sigma_{(2)}=\left(\begin{array}{c}
-\frac{a}{4} \leq x_{1} \leq 0 \\
x_{2}=\frac{b}{2} \\
-\frac{d}{2} \leq x_{3} \leq \frac{d}{2}
\end{array}\right), \quad \Sigma_{(3)}=\left(\begin{array}{c}
x_{1}=0 \\
-\frac{b}{2} \leq x_{2} \leq \frac{b}{2} \\
-\frac{d}{2} \leq x_{3} \leq \frac{d}{2}
\end{array}\right), \\
\Sigma_{(4)}=\left(\begin{array}{c}
-\frac{a}{4} \leq x_{1} \leq 0 \\
x_{2}=-\frac{b}{2} \\
-\frac{d}{2} \leq x_{3} \leq \frac{d}{2}
\end{array}\right), \quad \Sigma_{(5)}=\left(\begin{array}{c}
0 \leq x_{1} \leq \frac{a}{4} \\
x_{2}=-\frac{b}{2} \\
d \\
-\frac{d}{2} \leq x_{3} \leq \frac{d}{2}
\end{array}\right) .
\end{gathered}
$$

The interface $\Sigma_{k}$ defines the contact area of the two blocks $b^{A}, b^{B}$. Assuming linear stress distributions at the interface $\Sigma_{k}$ of the elementary cell, we can substitute the stresses with punctual forces and moments at the centre of area of each interface. Let $b^{A}, b^{B}$ be the two blocks interacting through interface $\Sigma_{k}$ and $\mathbf{F}_{(k)}^{b^{A}}\left(\right.$ resp. $\left.\mathbf{F}_{(k)}^{b^{B}}\right)$ and $\mathbf{M}_{(k)}^{b^{A}}$ (resp. $\left.\mathbf{M}_{(k)}^{b^{B}}\right)$ the force and the moment exerted by block $b^{B}$ over $b^{A}$ (resp. $b^{A}$ over $b^{B}$ ). This set of interaction forces and moments is self-balanced and is simply expressed as follows:

$$
\begin{gathered}
\mathbf{F}_{(k)}^{b}=\mathcal{K}_{(k)} \Delta \mathbf{u}_{(k)}^{b} \\
\mathbf{M}_{(k)}^{b}=C_{(k)} \Delta \boldsymbol{\omega}_{(k)}^{b}
\end{gathered}
$$

where the superscripts $b^{A}$ and $b^{B}$ have been simply replaced by $b$.

In equation (2), the expression of $\mathrm{K}_{(k)}$ and $\mathrm{C}_{(k)}$ is given by: 


$$
\begin{gathered}
\mathcal{K}_{(k)}=\left\{\begin{array}{l}
A_{H}\left(\begin{array}{ccc}
c_{Q} & 0 & 0 \\
0 & c_{N} & 0 \\
0 & 0 & c_{Q}
\end{array}\right), \text { for } k=1,2,4,5 \\
A_{V}\left(\begin{array}{ccc}
c_{N} & 0 & 0 \\
0 & c_{Q} & 0 \\
0 & 0 & c_{Q}
\end{array}\right), \text { for } k=3
\end{array} C_{(k)}=\left\{\begin{array}{l}
A_{H}\left(\begin{array}{ccc}
c_{M H 1} & 0 & 0 \\
0 & c_{M H 2} & 0 \\
0 & 0 & c_{M H 3}
\end{array}\right), \text { for } k=1,2,4,5 \\
A_{V}\left(\begin{array}{ccc}
c_{M V 1} & 0 & 0 \\
0 & c_{M V 2} & 0 \\
0 & 0 & c_{M V 3}
\end{array}\right), \text { for } k=3
\end{array},\right.\right.
\end{gathered}
$$

where $A_{H}\left(A_{V}\right)$ is the area of the horizontal (vertical) interface inside the elementary cell, $c_{M H i}\left(c_{M V i}\right)$ the bending stiffness of the horizontal (vertical) interface, $c_{N}$ the normal- and $c_{Q}$ the shear- elastic stiffness of the interface with dimensions $\quad \frac{[\mathrm{F}]}{[\mathrm{L}]^{3}} \quad$ and $\quad A_{H}=\frac{1}{4} a d, \quad A_{V}=b d, \quad c_{M H 1}=\frac{1}{12} c_{N} d^{2}$, $c_{M H 2}=\frac{1}{12} c_{Q}\left[\left(\frac{a}{4}\right)^{2}+d^{2}\right]$,

$c_{M H 3}=\frac{1}{12} c_{N}\left(\frac{a}{4}\right)^{2}, c_{M V 1}=\frac{1}{12} c_{Q}\left(b^{2}+d^{2}\right), c_{M V 2}=\frac{1}{12} c_{N} d^{2}, c_{M V 3}=\frac{1}{12} c_{N} b^{2}$.

$\Delta \mathbf{u}_{(k)}^{b}$ and $\Delta \boldsymbol{\omega}_{(k)}^{b}$ express accordingly the relative displacement and relative rotation at the centre of the area of interface $k$. For example, $\Delta \mathbf{u}_{(2)}^{b}=\mathbf{u}_{\mathrm{I}, \mathrm{J}+1}^{b}\left(\mathbf{r}_{(2)}\right)-\mathbf{u}_{\mathrm{I}-1, \mathrm{~J}}^{b}\left(\mathbf{r}_{(2)}\right)$, where $\mathbf{r}_{(2)}$ is the position vector of the center of interface $k=2$. If $\mathbf{r}_{\mathbf{c m}}$ is the position vector of the center of mass of the elementary cell then $\mathbf{r}_{(2)}-\mathbf{r}_{\mathrm{cm}}=\left\{-\frac{a}{4}, \frac{b}{2}, 0\right\}$. 


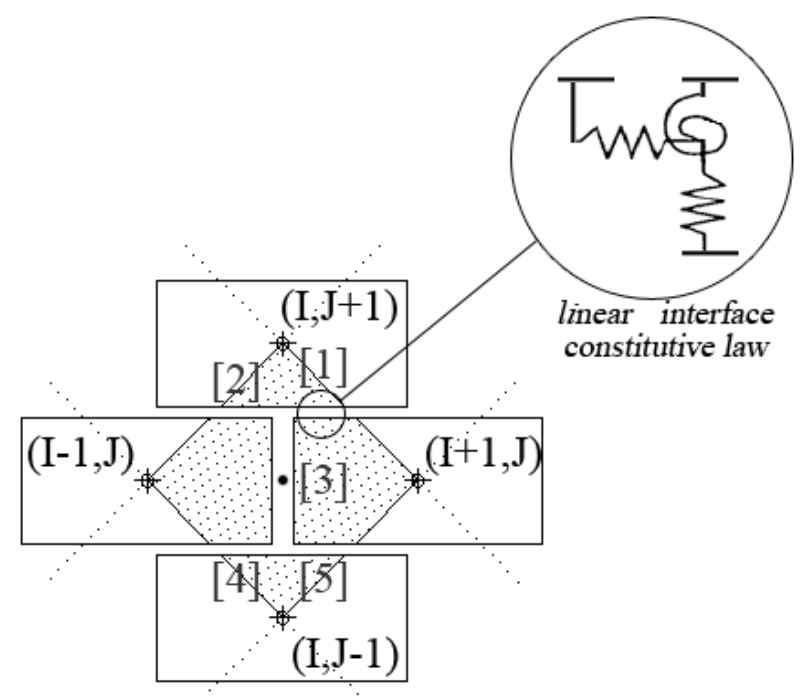

Figure 3. Numbering of the interfaces and blocks inside the elementary cell. The solid dot at the center of the elementary cell denotes the center mass of the elementary cell.

\section{Energy of the elementary cell}

For rigid blocks, the energy is only stored at the interfaces. Therefore the internal elastic energy per unit volume is:

$$
\mathcal{U}_{l a t}=\frac{1}{V}\left(\sum_{k=1}^{5} \frac{1}{2} \mathbf{F}_{(k)}^{b} \Delta \mathbf{u}_{(k)}^{b}+\sum_{k=1}^{5} \frac{1}{2} \mathbf{M}_{(k)}^{b} \Delta \boldsymbol{\omega}_{(k)}^{b}\right)
$$

The kinetic energy of the elementary cell is:

$$
\mathcal{T}_{\text {lat }}=\frac{1}{V}\left(\sum w_{\mathrm{I}, \mathrm{J}} \mathcal{T}_{\mathrm{I}, \mathrm{J}}\right)
$$

where $V$ is the volume of the elementary cell, $\mathcal{T}_{\mathrm{I}, \mathrm{J}}$ the kinetic energy of block $(\mathrm{I}, \mathrm{J})$ and $w_{\mathrm{I}, \mathrm{J}}$ is the weight of contribution of block (I,J) to the kinetic energy of the elementary cell $\mathcal{T}_{\text {lat }}$. The sum refers to the blocks that belong to the elementary cell.

$$
\mathcal{T}_{\mathrm{I}, \mathrm{J}}=\frac{1}{2} m\left(\dot{\mathbf{u}}_{\mathrm{I}, \mathrm{J}}^{b}\right)^{\mathrm{T}} \dot{\mathbf{u}}_{\mathrm{I}, \mathrm{J}}^{b}+\frac{1}{2}\left(\dot{\boldsymbol{\omega}}_{\mathrm{I}, \mathrm{J}}^{b}\right)^{\mathrm{T}} \mathbf{J} \dot{\boldsymbol{\omega}}_{\mathrm{I}, \mathrm{J}}^{b}
$$


where $m$ is the mass of a block and $\mathbf{J}$ is its inertia tensor expressed in its principal axes:

$$
\mathbf{J}=\left(\begin{array}{ccc}
J_{1} & 0 & 0 \\
0 & J_{2} & 0 \\
0 & 0 & J_{3}
\end{array}\right)=\left(\begin{array}{ccc}
\frac{1}{12} m\left(b^{2}+d^{2}\right) & 0 & 0 \\
0 & \frac{1}{12} m\left(a^{2}+d^{2}\right) & 0 \\
0 & 0 & \frac{1}{12} m\left(a^{2}+b^{2}\right)
\end{array}\right)
$$

\section{Equations of motion}

Using D'Alembert's principle we derive the equations of motion for each individual block of the masonry structure. These equations involve the six degrees of freedom of the nodes of the lattice model and they can be solved to give the mechanical response of the masonry wall. In Appendix we summarize these equations.

\section{The continuum}

There is a long standing argument whether matter is discrete or continuous, which, though, is not going to be resolved here. In the late $5^{\text {th }}$ century BC, Democritus and Leucippus supporting atomism and later Aristotle to reject their theory were perhaps the first philosophers to bring face to face the two different approaches. Obviously, masonry is a discrete medium as it is composed of basic building blocks, its "atoms", but integrating the equations of motion for each building block of a real structure is a computationally laborious task. Starting from the micro-scale of a masonry wall we will try in this section to bridge the Democritian and Aristotelian approach and formulate an equivalent Cosserat continuum that will be able to describe the three dimensional mechanical behavior of masonry walls. Averaging and homogenization are both techniques that are used for this passage from the discrete to continuum. 


\section{Homogenization based on the elastic energy}

The additional rotational degrees of freedom of the Cosserat continuum make it suitable for describing materials with internal structure. The homogenization procedure of a masonry structure as a Cosserat continuum is based on the construction of a continuum, which, for any 'virtual' translational- and rotationalfield, stores the same elastic energy as the corresponding lattice structure.

In the previous section, the elastic energy of the elementary cell of the masonry lattice model has been calculated. Accordingly, the average elastic energy of the Cosserat continuum over the volume $V$ of this elementary cell is set equal to that of the lattice cell (Askar 1968, [40]):

$$
\left\langle\mathcal{U}_{c}\right\rangle=\frac{1}{V} \int_{V} \mathcal{U}_{c} d V \approx \mathcal{U}_{c}\left(\mathbf{r}_{\mathrm{cm}}\right) \equiv \mathcal{U}_{\text {lat }}
$$

where $\mathcal{U}_{c}$ is the elastic energy density of the Cosserat continuum and $\mathcal{U}_{c}\left(\mathbf{r}_{\mathrm{cm}}\right)$ is its value at the center mass $\mathbf{r}_{\mathbf{c m}}=\left\{x_{1 \mathrm{~cm}}, x_{2 \mathrm{~cm}}\right\}$ of the elementary cell. $\mathcal{U}_{\text {lat }}$ is the elastic energy density of the elementary cell of the lattice model.

Let $\mathbf{u}^{c f}=\mathbf{u}^{c f}\left(x_{1}, x_{2}\right)$ and $\boldsymbol{\omega}^{c f}=\boldsymbol{\omega}^{c f}\left(x_{1}, x_{2}\right)$ be $\mathrm{C}^{2}$ translational and rotational fields, such that their values are identical to those of the displacements and rotations at the nodes of the lattice model (Figure 3):

$$
\begin{array}{l:l}
\mathbf{u}_{\mathrm{I}, \mathrm{J}}^{b} \equiv \mathbf{u}^{c f}\left(x_{1 c m}, x_{2 c m}\right) & \boldsymbol{\omega}_{\mathrm{I}, \mathrm{J}}^{b} \equiv \boldsymbol{\omega}^{c f}\left(x_{1 c m}, x_{2 c m}\right) \\
\mathbf{u}_{\mathrm{I}+1, \mathrm{~J}}^{b} \equiv \mathbf{u}^{c f}\left(x_{1 c m}+\frac{1}{2} a, x_{2 c m}\right) & \boldsymbol{\omega}_{\mathrm{I}+1, \mathrm{~J}}^{b} \equiv \boldsymbol{\omega}^{c f}\left(x_{1 c m}+\frac{1}{2} a, x_{2 c m}\right) \\
\mathbf{u}_{\mathrm{I}-1, \mathrm{~J}}^{b} \equiv \mathbf{u}^{c f}\left(x_{1 c m}-\frac{1}{2} a, x_{2 c m}\right) & \boldsymbol{\omega}_{\mathrm{I}-1, \mathrm{~J}}^{b} \equiv \boldsymbol{\omega}^{c f}\left(x_{1 c m}-\frac{1}{2} a, x_{2 c m}\right) \\
\mathbf{u}_{\mathrm{I}, \mathrm{J}+1}^{b} \equiv \mathbf{u}^{c f}\left(x_{1 c m}, x_{2 c m}+b\right) & \boldsymbol{\omega}_{\mathrm{I}, \mathrm{J}+1}^{b} \equiv \boldsymbol{\omega}^{c f}\left(x_{1 c m}, x_{2 c m}+b\right) \\
\mathbf{u}_{\mathrm{I}, \mathrm{J}-1}^{b} \equiv \mathbf{u}^{c f}\left(x_{1 c m}, x_{2 c m}-b\right) & \boldsymbol{\omega}_{\mathrm{I}, \mathrm{J}-1} \equiv \boldsymbol{\omega}^{c f}\left(x_{1 c m}, x_{2 c m}-b\right)
\end{array}
$$

On the other hand, each material point of a three-dimensional Cosserat continuum has three translational degrees of freedom $\mathbf{u}$ and three rotational degrees of freedom $\boldsymbol{\omega}^{c}$. The index " $c$ " is used to distinguish the Cosserat rotation from the local rigid-body rotation that derives from the Cosserat displacement field $\mathbf{u}$ : 


$$
\omega_{i j}=\frac{1}{2}\left(u_{i, j}-u_{j, i}\right) ;(.)_{, i}=\frac{\partial(.)}{\partial x_{i}} \quad(i=1,2,3)
$$

For the formulation of the constitutive relationships we need deformation measures, that are invariant to rigid body motions; i.e. the infinitesimal strain tensor:

$$
\varepsilon_{i j}=\frac{1}{2}\left(u_{i, j}+u_{j, i}\right)
$$

and the curvature tensor:

$$
\kappa_{i j}=\frac{\partial \omega_{i}^{c}}{\partial x_{j}}
$$

The equations (8), (9) and (10) are combined to give the following components of the so-called relative deformation tensor,

$$
\begin{array}{l|l|l|l}
\gamma_{11}=\frac{\partial u_{1}}{\partial x_{1}} & \gamma_{12}=\frac{\partial u_{1}}{\partial x_{2}}+\omega_{3}^{c} & \gamma_{13}=\frac{\partial u_{1}}{\partial x_{3}}-\omega_{2}^{c} & \gamma_{23}=\frac{\partial u_{2}}{\partial x_{3}}+\omega_{1}^{c} \\
\gamma_{22}=\frac{\partial u_{2}}{\partial x_{2}} & \gamma_{21}=\frac{\partial u_{2}}{\partial x_{1}}-\omega_{3}^{c} & \gamma_{31}=\frac{\partial u_{3}}{\partial x_{1}}+\omega_{2}^{c} & \gamma_{32}=\frac{\partial u_{3}}{\partial x_{2}}-\omega_{1}^{c} \\
\gamma_{33}=\frac{\partial u_{3}}{\partial x_{3}} & &
\end{array}
$$

Although in our case the wall spans only in directions $x_{1}$ and $x_{2}$ and the derivatives over $x_{3}$ direction are zero, for completeness and for presenting the general method followed here we keep the full deformation tensor. Moreover, the above general formulation of the deformation and curvature tensors would be necessary for the modelling of multi-layered masonry walls.

The 18 deformation measures, Eqs. (10) and (11), are conjugate in energy [35] to 18 stress measures: the 9 components of the non-symmetric stress tensor $\sigma_{i j}$ that are conjugate to the non-symmetric deformation tensor $\gamma_{i j}$ and the 9 couple stresses $m_{i j}$, which are conjugate with the 9 components of the curvature tensor $\kappa_{i j}$. 
The following dimensionless quantities are introduced:

$$
\begin{gathered}
\hat{a}=a / L, \quad \hat{b}=b / L, \quad \hat{d}=d / L, \quad \hat{c}_{N}=c_{N} / \rho g, \quad \hat{c}_{Q}=c_{Q} / \rho g, \quad \tau=\sqrt{\frac{g}{L}} t, \\
\hat{u}_{i}=u_{i} / L, \quad \hat{\gamma}_{i j}=\gamma_{i j}, \quad \hat{\kappa}_{i j}=\kappa_{i j} L, \quad \hat{\sigma}_{i j}=\sigma_{i j} / \rho g L, \quad \hat{m}_{i j}=m_{i j} / \rho g L^{2}
\end{gathered}
$$

where $\rho$ is the density of the material of the blocks and $L$ the size of the overall structure (i.e. the maximum distance between two points). When the size of the structure is big as compared to the size of the block, $\hat{a}, \hat{b}$ and $\hat{d}$ are small quantities. $\hat{\mathbf{r}}_{\mathbf{c m}}=\left\{\hat{x}_{\mathrm{r} m}, \hat{x}_{2 m}\right\} \in[0,1] \times[0,1]$.

The continuous fields $\hat{\mathbf{u}}^{c f}$ and $\boldsymbol{\omega}^{c f}$ can then be developed in Taylor series around $\hat{\mathbf{r}}_{c m}$ and up to the $2^{\text {nd }}$ and $1^{\text {st }}$ order respectively:

$$
\begin{aligned}
\hat{\mathbf{u}}^{c f}\left(\hat{x}_{\mathrm{k} m} \pm \frac{\hat{a}}{2}, \hat{x}_{2 m}\right) & \approx \hat{\mathbf{u}}^{c f}\left(\hat{\mathbf{r}}_{\mathrm{cm}}\right) \pm\left.\frac{\hat{a}}{2} \frac{\partial \hat{\mathbf{u}}^{c f}}{\partial \hat{x}_{1}}\right|_{\hat{r}_{\mathrm{rm}}}+\left.\frac{1}{2} \frac{\hat{a}^{2}}{4} \frac{\partial^{2} \hat{\mathbf{u}}^{c f}}{\partial \hat{x}_{1}^{2}}\right|_{\hat{r}_{\mathrm{cm}}} \\
\boldsymbol{\omega}^{c f}\left(\hat{x}_{\mathrm{r} m} \pm \frac{\hat{a}}{2}, \hat{x}_{2 m}\right) & \approx \boldsymbol{\omega}^{c f}\left(\hat{\mathbf{r}}_{\mathrm{cm}}\right) \pm\left.\frac{\hat{a}}{2} \frac{\partial \boldsymbol{\omega}^{c f}}{\partial \hat{x}_{1}}\right|_{\hat{r}_{\mathrm{cm}}} \\
\hat{\mathbf{u}}^{c f}\left(\hat{x}_{\mathrm{r} m}, \hat{x}_{2 m} \pm \hat{b}\right) & \approx \hat{\mathbf{u}}^{c f}\left(\hat{\mathbf{r}}_{\mathrm{cm}}\right) \pm\left.\hat{b} \frac{\partial \hat{\mathbf{u}}^{c f}}{\partial \hat{x}_{2}}\right|_{\hat{\mathbf{r}}_{\mathrm{cm}}}+\left.\frac{1}{2} \hat{b}^{2} \frac{\partial^{2} \hat{\mathbf{u}}^{c f}}{\partial \hat{x}_{1}^{2}}\right|_{\hat{\mathbf{r}}_{\mathrm{cm}}} \\
\boldsymbol{\omega}^{c f}\left(\hat{x}_{\mathrm{r} m}, \hat{x}_{2 m} \pm \hat{b}\right) & \approx \boldsymbol{\omega}^{c f}\left(\hat{\mathbf{r}}_{\mathrm{cm}}\right) \pm\left.\hat{b} \frac{\partial \boldsymbol{\omega}^{c f}}{\partial \hat{x}_{2}}\right|_{\hat{\mathbf{r}}_{\mathrm{cm}}}
\end{aligned}
$$

Notice that the rotational field is developed to one order lower than the translational field. Keeping the second order terms of the Taylor expansion for the rotations would introduce additional terms in the constitutive equations of the homogenised medium, which are two orders of magnitude smaller $\left(L^{-2}\right)$ than the ones kept to obtain the Cosserat continuum.

Substituting the discrete quantities with the continuous ones and setting $\mathbf{u}^{c f} \equiv \mathbf{u}, \quad \boldsymbol{\omega}^{c f} \equiv \boldsymbol{\omega}^{c}$ one can derive the elastic energy density of the elementary cell in terms of deformation measures: 


$$
\begin{aligned}
& \hat{\mathcal{U}}_{\text {lat }}=\frac{1}{2} \frac{\hat{A}_{H} \hat{a}}{\hat{b} \hat{d}}\left(\hat{c}_{N} \hat{\gamma}_{21}^{2}+\hat{c}_{Q}\left(\hat{\gamma}_{11}^{2}+\hat{\gamma}_{31}^{2}\right)\right)+2 \frac{\hat{A}_{H} \hat{b}}{\hat{a} \hat{d}}\left(\hat{c}_{N} \hat{\gamma}_{22}^{2}+\hat{c}_{Q}\left(\hat{\gamma}_{12}^{2}+\hat{\gamma}_{32}^{2}\right)\right) \\
& +\frac{1}{2} \frac{\hat{A}_{V} \hat{a}}{\hat{b} \hat{d}}\left(\hat{c}_{N} \hat{\gamma}_{11}^{2}+\hat{c}_{Q}\left(\hat{\gamma}_{21}^{2}+\hat{\gamma}_{31}^{2}\right)\right) \\
& +\frac{1}{8} \frac{\hat{a}}{\hat{b} \hat{d}}\left(4 \hat{A}_{V} \hat{c}_{M V 1}+A_{H}\left(\hat{c}_{Q} \hat{b}^{2}+4 \hat{c}_{M H 1}\right)\right) \hat{\kappa}_{11}^{2}+\frac{1}{8} \frac{\hat{a} \hat{b}}{\hat{d}} \hat{A}_{H} \hat{c}_{Q} \hat{\kappa}_{11} \hat{\kappa}_{22} \\
& +\frac{1}{32} \frac{\hat{b}}{\hat{a} \hat{d}} \hat{A}_{H}\left(\hat{c}_{Q} \hat{a}^{2}+64 \hat{c}_{M H 2}\right) \hat{\kappa}_{22}^{2}+2 \frac{b}{\hat{a} \hat{d}} \hat{A}_{H} \hat{c}_{M H 1} \hat{\kappa}_{12}^{2}+\frac{1}{32} \frac{\hat{b}}{\hat{a} \hat{d}} \hat{A}_{H}\left(\hat{c}_{N} \hat{a}^{2}+64 \hat{c}_{M H 3}\right) \hat{\kappa}_{32}^{2} \\
& +\frac{1}{128} \frac{\hat{a}}{\hat{b} \hat{d}}\left(64 \hat{A}_{V} \hat{c}_{M V 2}+\hat{A}_{H}\left(\hat{c}_{Q} \hat{a}^{2}+64 \hat{c}_{M H 2}\right)\right) \hat{\kappa}_{21}^{2} \\
& +\frac{1}{128} \frac{\hat{a}}{\hat{b} \hat{d}}\left(64 \hat{A}_{V} \hat{c}_{M V 3}+\hat{A}_{H}\left(\hat{c}_{N} \hat{a}^{2}+64 \hat{c}_{M H 3}+16 \hat{c}_{Q} \hat{b}^{2}\right)\right) \hat{\kappa}_{31}^{2} \\
& +\frac{1}{32} \frac{\hat{A}_{H} \hat{a}}{\hat{b} \hat{d}} \hat{c}_{N} \hat{\kappa}_{13}\left(\hat{a}^{2} \hat{\gamma}_{21,1}-4 \hat{b}^{2} \hat{\gamma}_{22,2}\right)+\frac{1}{32} \frac{\hat{A}_{H} \hat{a}}{\hat{b} \hat{d}} \hat{c}_{Q} \hat{\kappa}_{12}\left(\hat{a}^{2} \hat{\gamma}_{31,1}-4 \hat{b}^{2} \hat{\gamma}_{32,2}\right) \\
& +\frac{1}{32} \frac{\hat{A}_{H} \hat{a}^{3}}{\hat{b} \hat{d}} \hat{c}_{Q} \hat{\gamma}_{11,1}^{2}+\frac{1}{32} \frac{\hat{A}_{H} \hat{a}^{3}}{\hat{b} \hat{d}} \hat{c}_{N} \hat{\gamma}_{21,1}^{2}+\frac{1}{32} \frac{\hat{A}_{H} \hat{a}^{3}}{\hat{b} \hat{d}} \hat{c}_{Q} \hat{\gamma}_{31,1}^{2} \\
& +\frac{1}{2} \frac{\hat{A}_{H} \hat{b}^{3}}{\hat{a} \hat{d}} \hat{c}_{Q} \hat{\gamma}_{12,2}^{2}+\frac{1}{2} \frac{\hat{A}_{H} \hat{b}^{3}}{\hat{a} \hat{d}} \hat{c}_{N} \hat{\gamma}_{22,2}^{2}+\frac{1}{2} \frac{\hat{A}_{H} \hat{b}^{3}}{\hat{a} \hat{d}} \hat{c}_{Q} \hat{\gamma}_{32,2}^{2} \\
& -\frac{1}{4} \frac{\hat{A}_{H} \hat{a} \hat{b}}{\hat{d}} \hat{c}_{Q} \hat{\gamma}_{12,2} \hat{\gamma}_{11,1}-\frac{1}{4} \frac{\hat{A}_{H} \hat{a} \hat{b}}{\hat{d}} \hat{c}_{N} \hat{\gamma}_{22,2} \hat{\gamma}_{21,1}-\frac{1}{4} \frac{\hat{A}_{H} \hat{a} \hat{b}}{\hat{d}} \hat{c}_{Q} \hat{\gamma}_{32,2} \hat{\gamma}_{31,1}
\end{aligned}
$$

Here, we emphasize the homogenization of a masonry structure with a Cosserat continuum. In a Cosserat continuum the kinematical quantities, which appear in the constitutive equations, are restricted to strains and curvatures. Therefore, strain gradients (e.g. $\gamma_{i j, k}$ ) are neglected in the expression of the elastic energy density. Notice, that this is not equivalent to neglecting all second order derivatives of the displacement field.

As it will be shown in the next section, the obtained Cosserat continuum gives a good representation of the masonry structure.

Neglecting the strain gradients, $\gamma_{i j, k}$, the elastic energy density of the elementary cell yields: 


$$
\begin{aligned}
& \hat{\mathcal{U}}_{\text {lat }}=\frac{1}{2} \frac{\hat{A}_{H} \hat{a}}{\hat{b} \hat{d}}\left(\hat{c}_{N} \hat{\gamma}_{21}^{2}+\hat{c}_{Q}\left(\hat{\gamma}_{11}^{2}+\hat{\gamma}_{31}^{2}\right)\right)+2 \frac{\hat{A}}{\hat{a} \hat{b}}\left(\hat{c}_{N} \hat{\gamma}_{22}^{2}+\hat{c}_{Q}\left(\hat{\gamma}_{12}^{2}+\hat{\gamma}_{32}^{2}\right)\right) \\
& +\frac{1}{2} \frac{\hat{A_{V}} \hat{a}}{\hat{b} \hat{d}}\left(\hat{c}_{N} \hat{\gamma}_{11}^{2}+\hat{c}_{Q}\left(\hat{\gamma}_{21}^{2}+\hat{\gamma}_{31}^{2}\right)\right) \\
& +\frac{1}{8} \frac{\hat{a}}{\hat{b} \hat{d}}\left(4 \hat{A}_{V} \hat{c}_{M V 1}+A_{H}\left(\hat{c}_{Q} \hat{b}^{2}+4 \hat{c}_{M H 1}\right)\right) \hat{\kappa}_{11}^{2}+\frac{1}{8} \frac{\hat{a} \hat{b}}{\hat{d}} \hat{A}_{H} \hat{c}_{Q} \hat{\kappa}_{11} \hat{\kappa}_{22} \\
& +\frac{1}{32} \frac{\hat{b}}{\hat{a} \hat{d}} \hat{A}_{H}\left(\hat{c}_{Q} \hat{a}^{2}+64 \hat{c}_{M H 2}\right) \hat{\kappa}_{22}^{2}+2 \frac{b}{\hat{a} \hat{d}} \hat{A}_{H} \hat{c}_{M H 1} \hat{\kappa}_{12}^{2}+\frac{1}{32} \frac{\hat{b}}{\hat{a} \hat{d}} \hat{A}_{H}\left(\hat{c}_{N} \hat{a}^{2}+64 \hat{c}_{M H 3}\right) \hat{\kappa}_{32}^{2} \\
& +\frac{1}{128} \frac{\hat{a}}{\hat{b} \hat{d}}\left(64 \hat{A}_{V} \hat{c}_{M V 2}+\hat{A}_{H}\left(\hat{c}_{Q} \hat{a}^{2}+64 \hat{c}_{M H 2}\right)\right) \hat{\kappa}_{21}^{2} \\
& +\frac{1}{128} \frac{\hat{a}}{\hat{b} \hat{d}}\left(64 \hat{A}_{V} \hat{c}_{M V 3}+\hat{A}_{H}\left(\hat{c}_{N} \hat{a}^{2}+64 \hat{c}_{M H 3}+16 \hat{c}_{Q} \hat{b}^{2}\right)\right) \hat{\kappa}_{31}^{2}
\end{aligned}
$$

The constitutive relations can be obtained directly by differentiating the elastic energy density (Figure 4):

$$
\hat{\sigma}_{i j}\left(\hat{r}_{c m}\right)=\frac{\partial \hat{\mathcal{U}}_{c}\left(\hat{r}_{c m}\right)}{\partial \hat{\gamma}_{i j}} \text { and } \hat{m}_{i j}\left(\hat{r}_{c m}\right)=\frac{\partial \hat{\mathcal{U}}_{c}\left(\hat{r}_{c m}\right)}{\partial \hat{\kappa}_{i j}}
$$

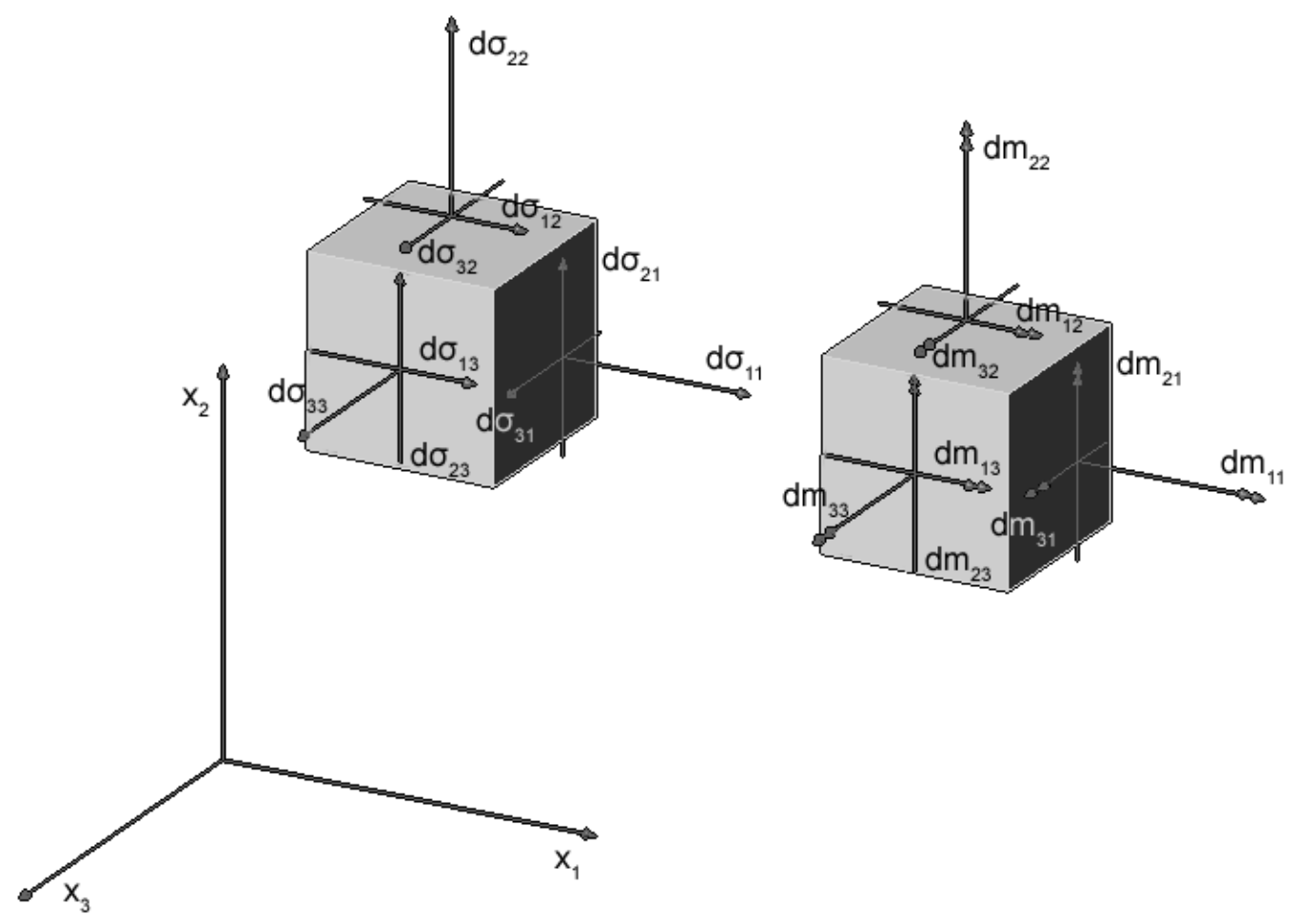

Figure 4. Stresses on element ( $\left.d x_{1}, d x 2, d x_{3}\right)$. 
Using equations (15) and (16), the constitutive equations of the equivalent elastic Cosserat continuum are obtained as:

$$
\begin{aligned}
& \hat{\sigma}_{11}=\left(\frac{\hat{c}_{Q} \hat{a}^{2}}{4 \hat{b}}+\hat{c}_{N} \hat{a}\right) \hat{\gamma}_{11} \\
& \hat{\sigma}_{22}=\hat{b} \hat{c}_{N} \hat{\gamma}_{22} \\
& \hat{\sigma}_{33}=0 \\
& \hat{\sigma}_{12}=\hat{b} \hat{c}_{Q} \hat{\gamma}_{12} \\
& \hat{\sigma}_{21}=\left(\frac{\hat{c}_{N} \hat{a}^{2}}{4 \hat{b}}+\hat{c}_{Q} \hat{a}\right) \hat{\gamma}_{21} \\
& \hat{\sigma}_{13}=0 \\
& \hat{\sigma}_{31}=\hat{c}_{Q} \frac{\hat{a}(\hat{a}+4 \hat{b})}{4 \hat{b}} \hat{\gamma}_{31} \\
& \hat{\sigma}_{23}=0 \\
& \hat{\sigma}_{32}=\hat{b} \hat{c}_{Q} \hat{\gamma}_{32}
\end{aligned}
$$

$$
\begin{aligned}
& \hat{m}_{11}=\frac{\left(4 \hat{b}\left(\hat{b}^{2}+\hat{d}^{2}\right) \hat{c}_{Q}+\hat{a}\left(\hat{c}_{N} \hat{d}^{2}+3 \hat{b}^{2} \hat{c}_{Q}\right)\right) \hat{a}}{48 \hat{b}} \hat{\kappa}_{11}+\frac{1}{32} \hat{b} \hat{a}^{2} \hat{c}_{Q} \hat{\kappa}_{22} \\
& \hat{m}_{22}=\frac{1}{32} \hat{b} \hat{a}^{2} \hat{c}_{Q} \hat{\kappa}_{11}+\frac{1}{48} \hat{b}\left(\hat{a}^{2}+4 \hat{d}^{2}\right) \hat{c}_{Q} \hat{\kappa}_{22} \\
& \hat{m}_{33}=0 \\
& \hat{m}_{12}=\frac{1}{12} \hat{b} \hat{d}^{2} \hat{c}_{N} \hat{\kappa}_{12} \\
& \hat{m}_{21}=\frac{\left(16 \hat{b} \hat{d}^{2} \hat{c}_{N}+\hat{a}\left(\hat{a}^{2}+4 \hat{d}^{2}\right) \hat{c}_{Q}\right) \hat{a}}{192 \hat{b}} \hat{\kappa}_{21} \\
& \hat{m}_{13}=0 \\
& \hat{m}_{31}=\frac{\hat{a}\left(\left(\hat{a}^{3}+16 \hat{b}^{3}\right) \hat{c}_{N}+12 \hat{a} \hat{b}^{2} \hat{c}_{Q}\right)}{192 \hat{b}} \hat{\kappa}_{31} \\
& \hat{m}_{23}=0 \\
& \hat{m}_{32}=\frac{1}{48} \hat{a}^{2} \hat{b} \hat{c}_{N} \hat{\kappa}_{32}
\end{aligned}
$$

In the obtained constitutive relationships (16), the stresses and the couple stresses are uncoupled. As shown by Trovalusci \& Masiani [9], this is a general result for 
centro-symmetric materials (the central symmetry is the material symmetry of any periodic assemblages of elements).

These equations show the anisotropic character of the equivalent continuum. This anisotropy is explained by the fact that each block has four neighbours in the $x_{2}$ direction and only two neighbours in the $x_{1}$ direction. Notice that in a Cosserat continuum the stress tensor is generally non-symmetric $\sigma_{i j} \neq \sigma_{j i}$.

\section{Inertia terms}

In the previous section the constitutive law of the Cosserat continuum has been derived from the elastic energy of the elementary cell of the lattice. Similarly, in this section the inertia terms of the continuous model are determined from the kinetic energy of the elementary cell of the lattice.

The kinetic energy of the Cosserat continuum is calculated over the volume $V$ of the elementary cell and is equated to the kinetic energy of the lattice cell:

$$
\left\langle\mathcal{T}_{c}\right\rangle=\frac{1}{V} \int_{V} \mathcal{T}_{c} d V \approx \mathcal{T}_{c}\left(r_{c m}\right) \equiv \mathcal{T}_{\text {lat }}
$$

where $\mathcal{T}_{c}$ is the kinetic energy of the Cosserat continuum, $\mathcal{T}_{c}\left(r_{c m}\right)$ is the kinetic energy of the Cosserat continuum calculated at the center of mass $r_{c m}$ of the elementary cell and $\mathcal{T}_{\text {lat }}$ is the kinetic energy of the elementary cell of the lattice model.

Substituting the discrete quantities of Eq.(5) with the continuous ones and setting $\mathbf{u}^{b} \equiv \mathbf{u}, \quad \boldsymbol{\omega}^{b} \equiv \boldsymbol{\omega}^{c}$ the kinetic energy of the elementary cell becomes:

$$
\begin{aligned}
& \mathcal{T}_{\text {lat }}=\frac{1}{2} \rho\left(\dot{u}_{1}^{2}+\dot{u}_{2}^{2}+\dot{u}_{3}^{2}\right)+\frac{1}{2}\left(\frac{J_{1}}{V} \dot{\omega}_{1}^{c 2}+\frac{J_{2}}{V} \dot{\omega}_{2}^{c 2}+\frac{J_{3}}{V} \dot{\omega}_{3}^{c 2}\right)+ \\
& \frac{1}{8} \rho b^{2}\left(\frac{\partial \dot{u}_{1}^{2}}{\partial x_{2}}+\frac{\partial \dot{u}_{2}^{2}}{\partial x_{2}}+\frac{\partial \dot{u}_{3}^{2}}{\partial x_{2}}\right)+\frac{1}{8} b^{2}\left(\frac{J_{1}}{V} \frac{\partial \dot{\omega}_{1}^{c^{2}}}{\partial x_{2}}+\frac{J_{2}}{V} \frac{\partial \dot{\omega}_{2}^{c^{2}}}{\partial x_{2}}+\frac{J_{3}}{V} \frac{\partial \dot{\omega}_{3}^{c^{2}}}{\partial x_{2}}\right)+ \\
& \frac{3}{32} \rho a^{2}\left(\frac{\partial \dot{u}_{1}^{2}}{\partial x_{1}}+\frac{\partial \dot{u}_{2}^{2}}{\partial x_{1}}+\frac{\partial \dot{u}_{3}^{2}}{\partial x_{1}}\right)+\frac{3}{32} a^{2}\left(\frac{J_{1}}{V} \frac{\partial \dot{\omega}_{1}^{c^{2}}}{\partial x_{1}}+\frac{J_{2}}{V} \frac{\partial \dot{\omega}_{2}^{c 2}}{\partial x_{1}}+\frac{J_{3}}{V} \frac{\partial \dot{\omega}_{3}^{c 2}}{\partial x_{1}}\right)
\end{aligned}
$$


The inertia terms $Q$ can now be determined:

$$
Q_{i}=\frac{\partial}{\partial t}\left(\frac{\partial \mathcal{T}_{c}\left(r_{c m}\right)}{\partial \dot{q}_{i}}\right)
$$

where $\dot{\mathbf{q}}=\left\{\dot{u}_{1}, \dot{u}_{2}, \dot{u}_{3}, \dot{\omega}_{1}^{c}, \dot{\omega}_{2}^{c}, \dot{\omega}_{3}^{c}\right\}$.

These terms are introduced into the dynamic equations of the Cosserat continuum:

$$
\begin{aligned}
& \hat{\sigma}_{11,1}+\hat{\sigma}_{12,2}+\hat{\sigma}_{13,3}-\hat{u}_{1}^{\prime \prime}=0 \\
& \hat{\sigma}_{21,1}+\hat{\sigma}_{22,2}+\hat{\sigma}_{23,3}-\hat{u}_{2}^{\prime \prime}=0 \\
& \hat{\sigma}_{31,1}+\hat{\sigma}_{32,2}+\hat{\sigma}_{33,3}-\hat{u}_{3}^{\prime \prime}=0 \\
& \hat{m}_{11,1}+\hat{m}_{12,2}+\hat{m}_{13,3}+\hat{\sigma}_{32}-\hat{\sigma}_{23}-\hat{J}_{1} \omega_{1}^{c^{\prime \prime}}=0 \\
& \hat{m}_{21,1}+\hat{m}_{22,2}+\hat{m}_{23,3}+\hat{\sigma}_{13}-\hat{\sigma}_{31}-\hat{J}_{2} \omega_{2}^{c^{\prime \prime}}=0 \\
& \hat{m}_{31,1}+\hat{m}_{32,2}+\hat{m}_{33,3}+\hat{\sigma}_{21}-\hat{\sigma}_{12}-\hat{J}_{3} \omega_{3}^{c \prime \prime}=0
\end{aligned}
$$

,where: (.)" $\equiv \frac{\partial^{2}(.)}{\partial \tau^{2}}$ and from Eqs. (12) and (20) we get that: $\hat{J}_{1}=\frac{1}{12}\left(\hat{b}^{2}+\hat{d}^{2}\right)$, $\hat{J}_{2}=\frac{1}{12}\left(\hat{a}^{2}+\hat{d}^{2}\right), \hat{J}_{3}=\frac{1}{12}\left(\hat{a}^{2}+\hat{b}^{2}\right)$.

\section{Dispersion function}

The domain of validity of the previous description of a masonry wall by a Cosserat continuum is evaluated by comparing its dynamic response with the dynamic response of the lattice model. The dynamic response of a structure is characterized by its dispersion function that relates the wave propagation frequency to the wavelength. For linear elastic behaviour it is possible to derive analytically the dispersion function of the lattice- and of the continuous systems by using discrete and continuous Fourier transforms respectively.

\section{Fourier transform of the Cosserat equations}

The Fourier transform of a function is defined as: 


$$
\mathrm{G}\left(\hat{k}_{1}, \hat{k}_{2}, \hat{\omega}\right)=\mathcal{F}\left\{\mathrm{g}\left(\hat{x}_{1}, \hat{x}_{2}, \tau\right)\right\}=\int_{-\infty}^{+\infty} \int_{-\infty}^{+\infty} \int_{-\infty}^{+\infty} \mathrm{g}\left(\hat{x}_{1}, \hat{x}_{2}, \tau\right) \mathrm{e}^{\mathrm{i}\left(\hat{k}_{1} \hat{x}_{1}+\hat{k}_{2} \hat{x}_{2}+\hat{\omega} \tau\right)} d \hat{x}_{1} d \hat{x}_{2} d \tau
$$

where $\mathrm{i}=\sqrt{-1}, \hat{k}_{1}$ and $\hat{k}_{2}$ the wave numbers at $\hat{x}_{1}$ and $\hat{x}_{2}$ direction, respectively, and $\hat{\omega}$ the frequency.

The inverse transform is:

$$
\mathrm{g}\left(\hat{x}_{1}, \hat{x}_{2}, \tau\right)=\mathcal{F}^{-1}\left\{\mathrm{G}\left(\hat{k}_{1}, \hat{k}_{2}, \hat{\omega}\right)\right\}=\int_{-\infty}^{+\infty} \int_{-\infty}^{+\infty} \int_{-\infty}^{+\infty} \mathrm{G}\left(\hat{k}_{1}, \hat{k}_{2}, \hat{\omega}\right) \mathrm{e}^{-\mathrm{i}\left(\hat{k}_{1} \hat{x}_{1}+\hat{k}_{2} \hat{x}_{2}+\hat{\omega} \tau\right)} d \hat{k}_{1} d \hat{k}_{2} d \hat{\omega}
$$

The Fourier transform will be denoted as:

$$
\mathrm{g}\left(\hat{x}_{1}, \hat{x}_{2}, \tau\right) \rightleftharpoons \mathrm{G}\left(\hat{k}_{1}, \hat{k}_{2}, \hat{\omega}\right)
$$

Introducing the constitutive relations (17) in the Cosserat dynamic equations (Eqs. (21)) and using Eqs. (12) we obtain the following partial differential equations for the displacement and Cosserat rotation fields:

$$
\begin{aligned}
& \hat{b} \hat{c}_{Q} \frac{\partial \omega_{3}^{c}}{\partial x_{2}}+\hat{b} \hat{c}_{Q} \frac{\partial^{2} \hat{u}_{1}}{\partial x_{2}{ }^{2}}+\left(\frac{\hat{c}_{Q} \hat{a}^{2}}{4 \hat{b}}+\hat{c}_{N} \hat{a}\right) \frac{\partial^{2} \hat{u}_{1}}{\partial x_{1}{ }^{2}}-\hat{u}_{1}^{\prime \prime}=0 \\
& \hat{b} \hat{c}_{N} \frac{\partial^{2} \hat{u}_{2}}{\partial x_{2}{ }^{2}}-\frac{\hat{a}\left(\hat{a} \hat{c}_{N}+4 \hat{b} \hat{c}_{Q}\right)}{4 \hat{b}} \frac{\partial \omega_{3}^{c}}{\partial x_{1}}+\left(\frac{\hat{c}_{N} \hat{a}^{2}}{4 \hat{b}}+\hat{c}_{Q} \hat{a}\right) \frac{\partial^{2} \hat{u}_{2}}{\partial x_{1}{ }^{2}}-\hat{u}_{2}^{\prime \prime}=0 \\
& \left(-\frac{\hat{c}_{N} \hat{a}^{2}}{4 \hat{b}}-\hat{c}_{Q} \hat{a}-\hat{b} \hat{c}_{Q}\right) \omega_{3}^{c}-\hat{b}_{\hat{c}_{Q}} \frac{\partial \hat{u}_{1}}{\partial x_{2}}+\frac{1}{48} \hat{a}^{2} \hat{b} \hat{c}_{N} \frac{\partial^{2} \omega_{3}^{c}}{\partial x_{2}{ }^{2}}+ \\
& +\left(\frac{\hat{c}_{N} \hat{a}^{2}}{4 \hat{b}}+\hat{c}_{Q} \hat{a}\right) \frac{\partial \hat{u}_{2}}{\partial x_{1}}+\frac{\hat{a}\left(\hat{a}^{3} \hat{c}_{N}+16 \hat{b}^{3} \hat{c}_{N}+12 \hat{a} \hat{b}^{2} \hat{c}_{Q}\right)}{192 \hat{b}} \frac{\partial^{2} \omega_{3}^{c}}{\partial x_{1}{ }^{2}}-\hat{J}_{3} \omega_{3}^{\prime \prime c}=0
\end{aligned}
$$




$$
\begin{gathered}
-\hat{b} \hat{c}_{Q} \frac{\partial \omega_{1}^{c}}{\partial x_{2}}+\hat{b} \hat{c}_{Q} \frac{\partial^{2} \hat{u}_{3}}{\partial x_{2}{ }^{2}}+\frac{\hat{a}(\hat{a}+4 \hat{b}) \hat{c}_{Q}}{4 \hat{b}} \frac{\partial \omega_{2}^{c}}{\partial x_{1}}+\frac{\hat{a}(\hat{a}+4 \hat{b}) \hat{c}_{Q}}{4 \hat{b}} \frac{\partial^{2} \hat{u}_{3}}{\partial x_{1}{ }^{2}}-\hat{u}_{3}^{\prime \prime}=0 \\
-\hat{b} \hat{c}_{Q} \omega_{1}^{c}+\hat{b} \hat{c}_{Q} \frac{\partial \hat{u}_{3}}{\partial x_{2}}+\frac{1}{12} \hat{b} \hat{d}^{2} \hat{c}_{N} \frac{\partial^{2} \omega_{1}^{c}}{\partial x_{2}{ }^{2}}+\frac{1}{32} \hat{a}^{2} \hat{b} \hat{c}_{Q} \frac{\partial^{2} \omega_{2}^{c}}{\partial x_{1} \partial x_{2}}+ \\
+\hat{a} \frac{4 \hat{b}\left(\hat{b}^{2}+\hat{d}^{2}\right) \hat{c}_{Q}+\hat{a}\left(\hat{d}^{2} \hat{c}_{N}+3 \hat{b}^{2} \hat{c}_{Q}\right)}{48 \hat{b}} \frac{\partial^{2} \omega_{1}^{c}}{\partial x_{1}^{2}}-\hat{J}_{1} \omega_{1}^{\prime \prime c}=0 \\
-\frac{\hat{a}(\hat{a}+4 \hat{b}) \hat{c}_{Q}}{4 \hat{b}} \omega_{2}^{c}+\frac{1}{48} \hat{b}\left(\hat{a}^{2}+4 \hat{d}^{2}\right) \hat{c}_{Q} \frac{\partial^{2} \omega_{2}^{c}}{\partial x_{2}^{2}}-\frac{\hat{a}(\hat{a}+4 \hat{b}) \hat{c}_{Q}}{4 \hat{b}} \frac{\partial \hat{u}_{3}}{\partial x_{1}}+ \\
+\frac{1}{32} \hat{a}^{2} \hat{b} \hat{c}_{Q} \frac{\partial^{2} \omega_{1}^{c}}{\partial x_{1} \partial x_{2}}+\hat{a} \frac{16 \hat{b} \hat{d}^{2} \hat{c}_{N}+\hat{a}\left(\hat{a}^{2}+4 \hat{d}^{2}\right) \hat{c}_{Q}}{192 \hat{b}} \frac{\partial^{2} \omega_{2}^{c}}{\partial x_{1}^{2}}-\hat{J}_{2} \omega_{2}^{\prime c c}=0
\end{gathered}
$$

These equations are separated into two uncoupled sets, Eqs. (25) and (26), which correspond to the in- and the out-of-plane deformation respectively. Therefore, the initial problem is finally separated in two independent problems, a "membrane"and a "plate" problem.

The Fourier transform of the above equations leads to a homogeneous linear system of equations. This system possesses non-trivial solutions when its determinant vanishes. The triads $\left(\hat{k}_{1}, \hat{k}_{2}, \hat{\omega}\right)$ that make the determinant zero define the six dispersion functions corresponding to the oscillation modes of the system. These oscillation modes are grouped into two sets; i.e. mode 2, 3 and 6 for in- and mode 1, 4 and 5 for out-of-plane deformation. For modes 1, 2 and 3 the amplitude of the displacement field is bigger than the amplitude of the rotational field, whereas the contrary is observed for modes 4, 5 and 6 . One could show that in case of an isotropic Cosserat continuum, modes 1, 2 and 3 are purely translational, whereas modes 4,5 and 6 are purely rotational. For an anisotropic Cosserat continuum the propagation of translational waves includes small rotations while the propagation of rotational waves includes small translations. 


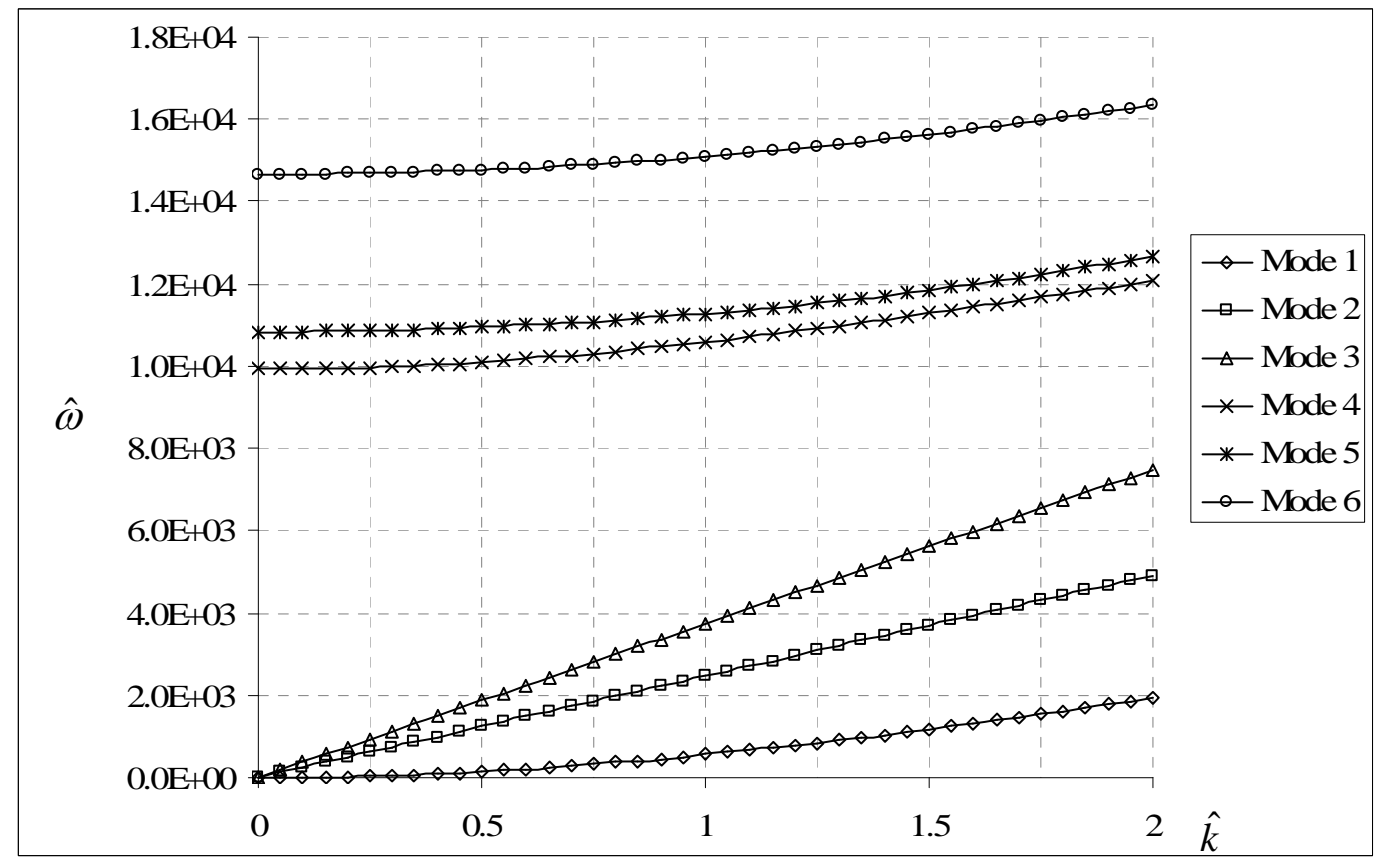

Figure 5. Cosserat model: Frequency $\hat{\omega}$ of the various oscillation modes for wave propagating at $45^{\circ}\left(\hat{k}_{1}=\frac{\sqrt{2}}{2} \hat{k}, \quad \hat{k}_{2}=\frac{\sqrt{2}}{2} \hat{k}\right)$.

In the numerical examples the dimensions of the blocks are $a=390 \mathrm{~mm}$, $b=190 \mathrm{~mm}$ and $d=190 \mathrm{~mm}$ and their specific weight $20 \mathrm{kN} / \mathrm{m}^{3}$. The thickness of the joints is $10 \mathrm{~mm}$. The Young's Modulus of the mortar is 4GPa and its Poisson's ratio 0.2. Consequently, the elastic normal- and shear-stiffness of the interfaces are $\hat{c}_{N}=2 \cdot 10^{7}, \hat{c}_{Q}=0.8 \cdot 10^{7}$.

\section{Fourier transform of the lattice equations}

A similar procedure is followed in order to derive the dispersion function for the lattice model. For the lattice system of equations (see Eqs. (33) at the Appendix) the function $g_{\mathrm{I} \pm n_{1}, \mathrm{~J} \pm n_{2}}(\tau)$ can be written as:

$$
g_{\mathrm{I} \pm n_{1}, \mathrm{~J} \pm n_{2}}(\tau)=\delta\left(x_{1} \mp \frac{1}{2} n_{1} a\right) \delta\left(x_{2} \mp n_{2} b\right) f\left(\hat{x}_{1}, \hat{x}_{2}, \tau\right)
$$

$\delta$ is the Dirac delta function. The discrete Fourier transform of function $g_{\mathrm{I}, \mathrm{J}}(\tau)$ is denoted as: 


$$
g_{\mathrm{I}, \mathrm{J}}(\tau) \rightleftharpoons G\left(\hat{k}_{1}, \hat{k}_{2}, \hat{\omega}\right)
$$

and consequently:

$$
g_{\mathrm{I} \pm n_{1}, \mathrm{~J} \pm n_{2}}(\tau) \rightleftharpoons e^{-i\left( \pm n_{1} \frac{1}{2} a \hat{k}_{1} \pm n_{2} b \hat{k}_{2}\right)} G\left(\hat{k}_{1}, \hat{k}_{2}, \hat{\omega}\right)
$$

Similarly to the continuous case, the dispersion function is determined by applying the Fourier transform to the dynamic equations of the lattice model. Again, modes 2, 3 and 6 correspond to the in- and modes 1, 4 and 5 to the out-of-plane deformation of the structure. For modes 1,2 and 3 translational waves dominate over rotational waves and for modes 4,5 and 6 the opposite is observed.

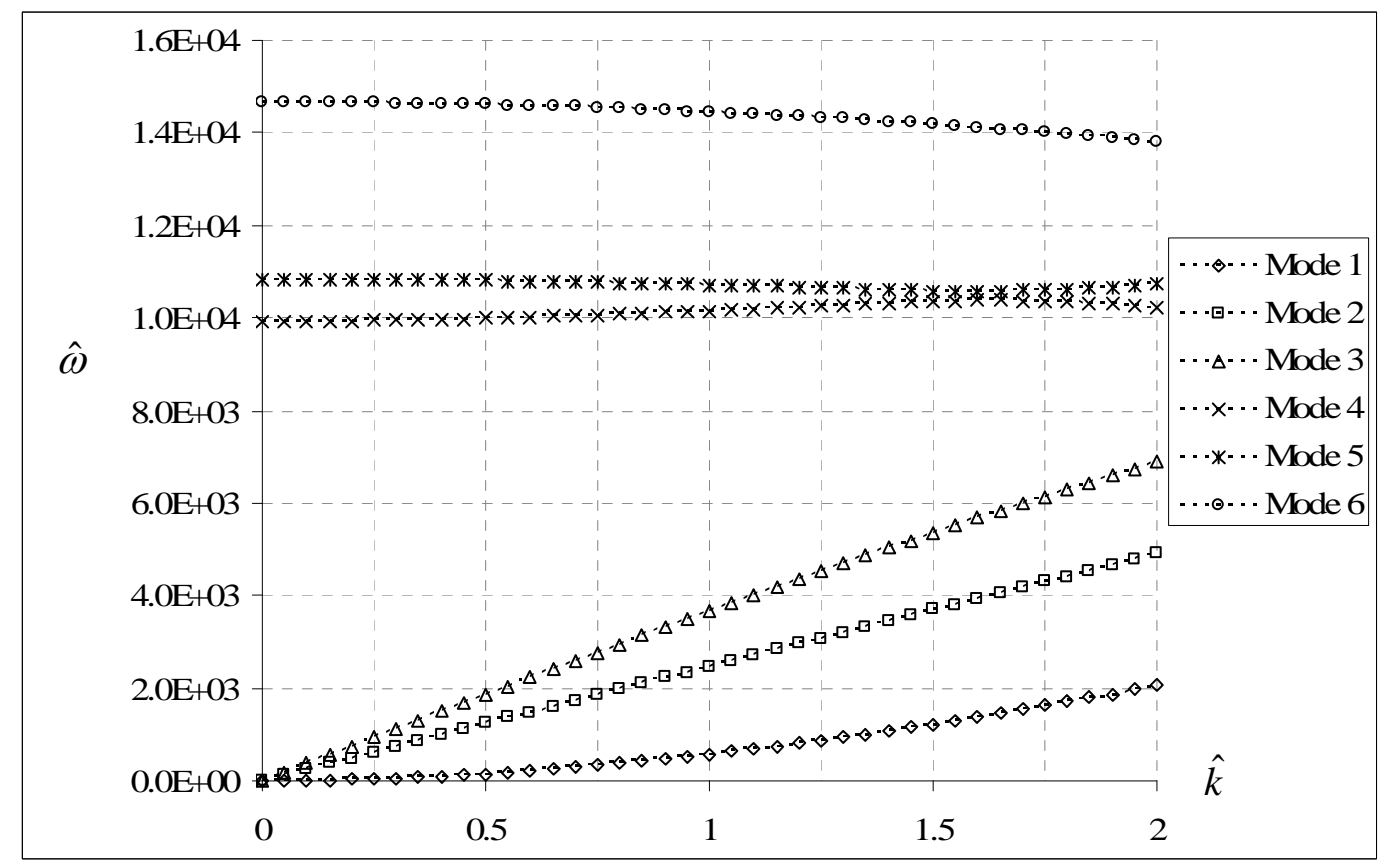

Figure 6. Lattice model: Frequency $\hat{\omega}$ of the various oscillation modes for wave propagating at $45^{\circ}\left(\hat{k}_{1}=\frac{\sqrt{2}}{2} \hat{k}, \quad \hat{k}_{2}=\frac{\sqrt{2}}{2} \hat{k}\right)$.

\section{Validation of the Cosserat model}

The dimensionless wave or phase velocity, which is the velocity with which planes of equal phase, crests or troughs, progress through the medium [41] is defined as: 


$$
\hat{v}=\frac{\hat{\omega}}{\|\hat{\mathbf{k}}\|}
$$

where $\|\cdot\|$ is the Euclidean norm, $\hat{\mathbf{k}}=\hat{k}\left(\cos \theta \mathbf{e}_{1}+\sin \theta \mathbf{e}_{2}\right), \theta$ the direction at which the wave is propagating and $\mathbf{e}_{j}$ the unit vectors of the reference system (Figure 4). Evidently, $\hat{k}_{1}=\hat{k} \cos \theta$ and $\hat{k}_{2}=\hat{k} \sin \theta$.

The wave length of the propagating wave normalized by the block dimension $\alpha$ is equal to:

$$
\hat{\lambda}=\frac{1}{\hat{\alpha}} \frac{2 \pi}{\hat{k}}
$$

The validation of the Cosserat model with the lattice one is carried out for propagating waves in direction $\theta$ and wave length $\hat{\lambda}$. For large wave-lengths $\hat{\lambda}$ the wave velocity modes 1,2 and 3 (translational waves) is finite, while the wave velocity of modes 4, 5 and 6 (rotational waves) tends to infinity. However, in this case the amplitudes of the rotational waves vanish. Therefore, for large wave lengths the Cosserat effects disappear and wave propagation in classical continuum is retrieved. On Figures 7 and 8 we plot the wave velocities for the various oscillation modes both for the Cosserat and the lattice model.

The comparison of the Cosserat model with the lattice model is carried out further in terms of the relative error $e_{\%}$ :

$$
e_{\%}=100 \frac{\left|\hat{v}_{C o s}-\hat{v}_{l a t}\right|}{\hat{v}_{l a t}}
$$

On Figures 9 and 10 and 11 we plot the relative error of the Cosserat model. 


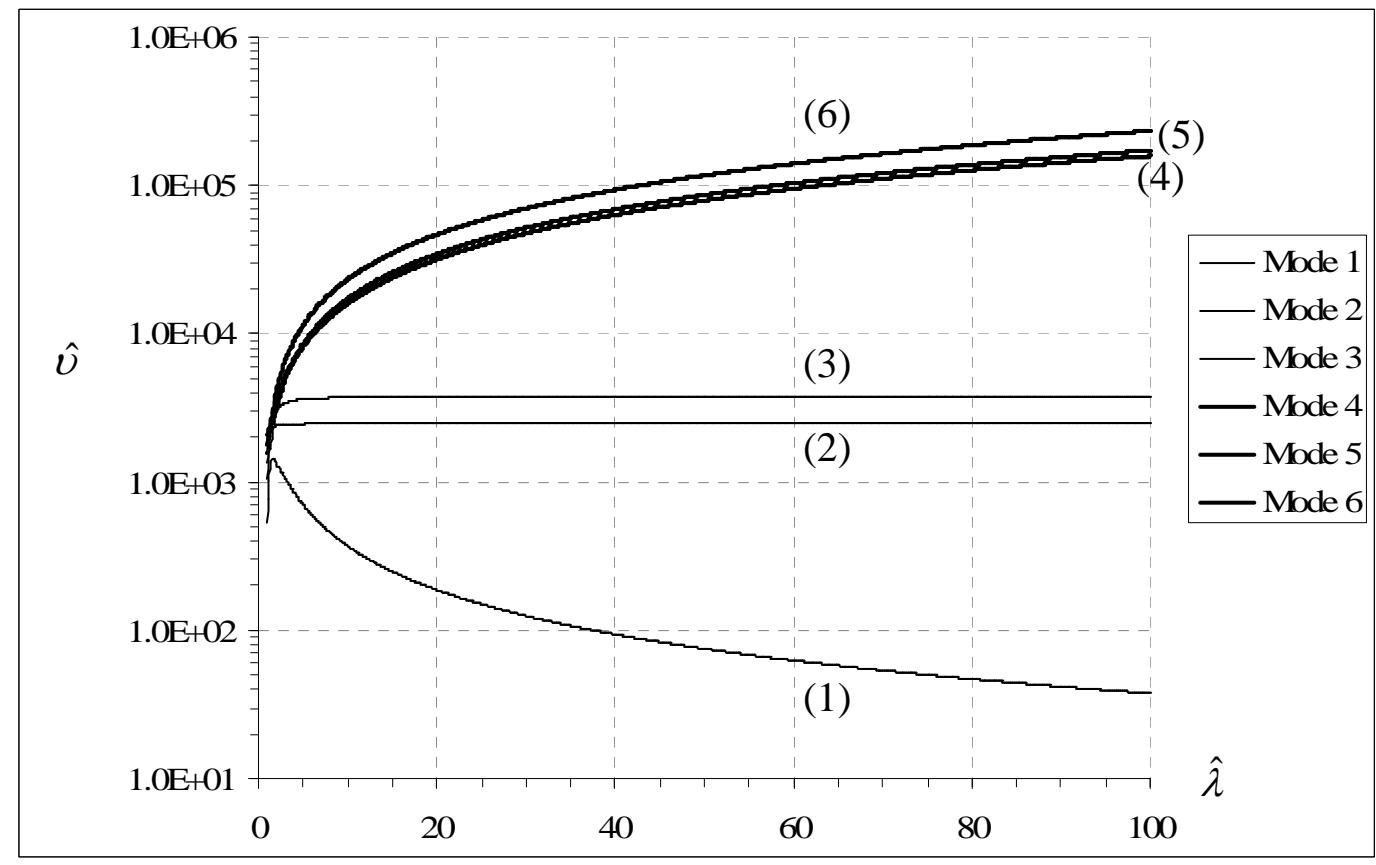

Figure 7. Lattice model: Distinction between oscillation modes 1, 2 and 3 (translational waves) and modes 4, 5 and 6 (rotational waves) in terms of the wave velocity $\hat{v}$ for wave propagating at $45^{\circ}\left(\hat{k}_{1}=\frac{\sqrt{2}}{2} \hat{k}, \quad \hat{k}_{2}=\frac{\sqrt{2}}{2} \hat{k}\right) . \hat{\lambda}$ is the wave length normalized by the block dimension $\alpha$.

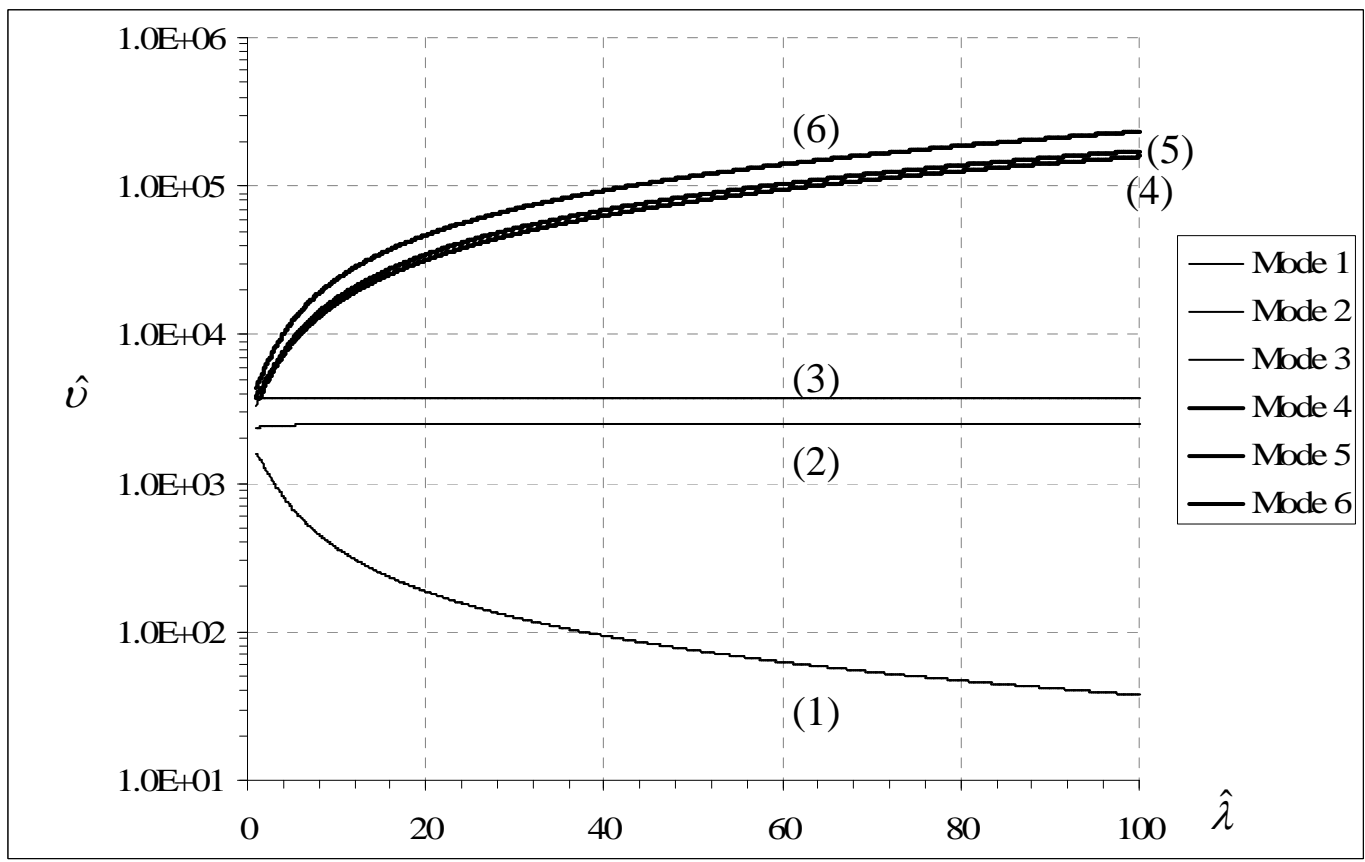

Figure 8. Cosserat model: Distinction between oscillation modes 1, 2 and 3 (translational waves) and modes 4, 5 and 6 (rotational waves) in terms of the wave velocity $\hat{v}$ for wave propagating at $45^{\circ}\left(\hat{k}_{1}=\frac{\sqrt{2}}{2} \hat{k}, \quad \hat{k}_{2}=\frac{\sqrt{2}}{2} \hat{k}\right) . \hat{\lambda}$ is the wave length normalized by the block dimension $\alpha$. 


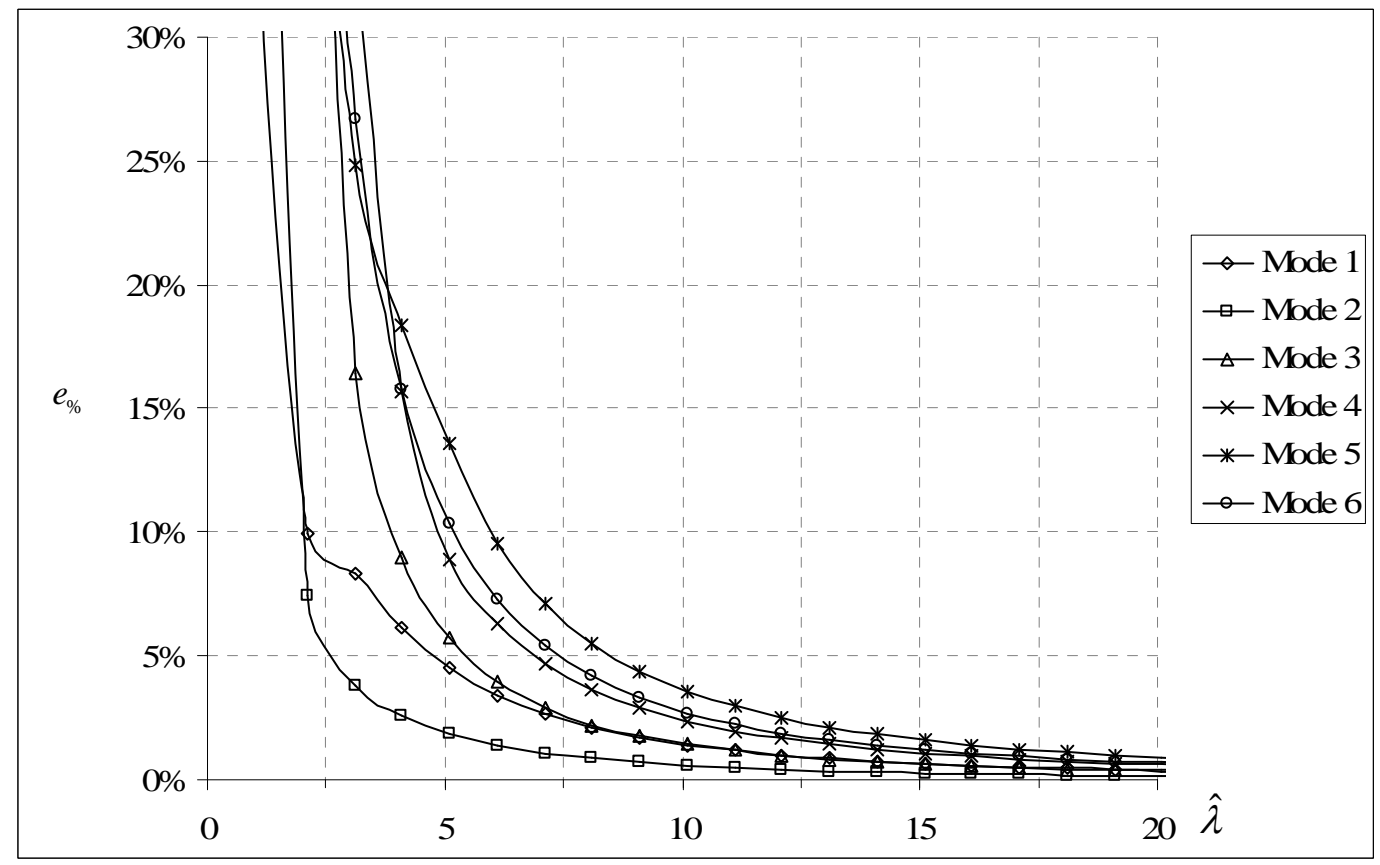

Figure 9. Cosserat versus Lattice: Relative error $e_{\%}$ of the various oscillation modes for wave propagating at $0^{\circ}\left(\hat{k}_{1}=\hat{k}, \quad \hat{k}_{2}=0\right) . \hat{\lambda}$ is the wave length normalized by the block dimension $\alpha$.

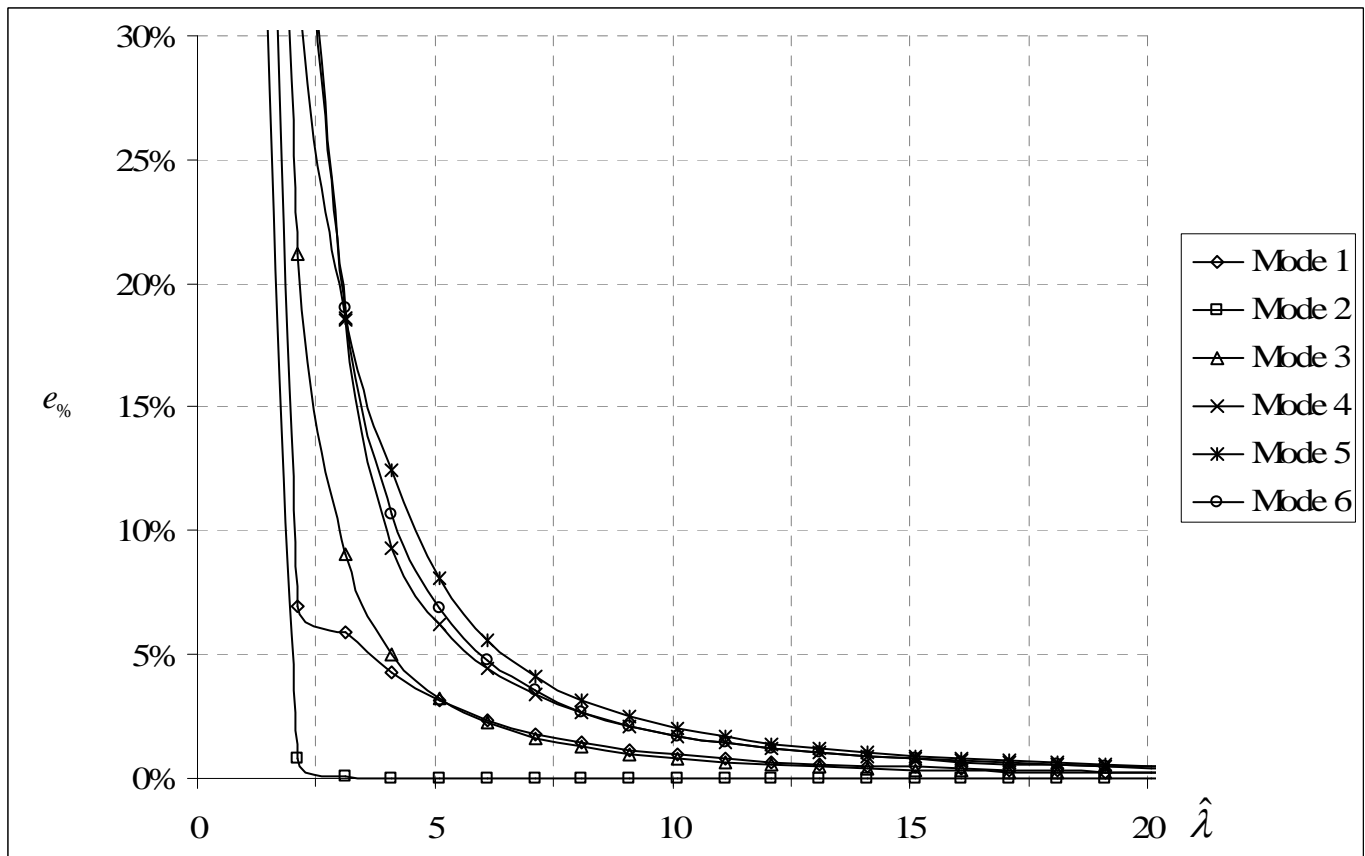

Figure 10. Cosserat versus Lattice: Relative error $e_{\%}$ of the various oscillation modes for wave propagating at $45^{\circ}\left(\hat{k}_{1}=\frac{\sqrt{2}}{2} \hat{k}, \quad \hat{k}_{2}=\frac{\sqrt{2}}{2} \hat{k}\right) \cdot \hat{\lambda}$ is the wave length normalized by the block dimension $\alpha$ 


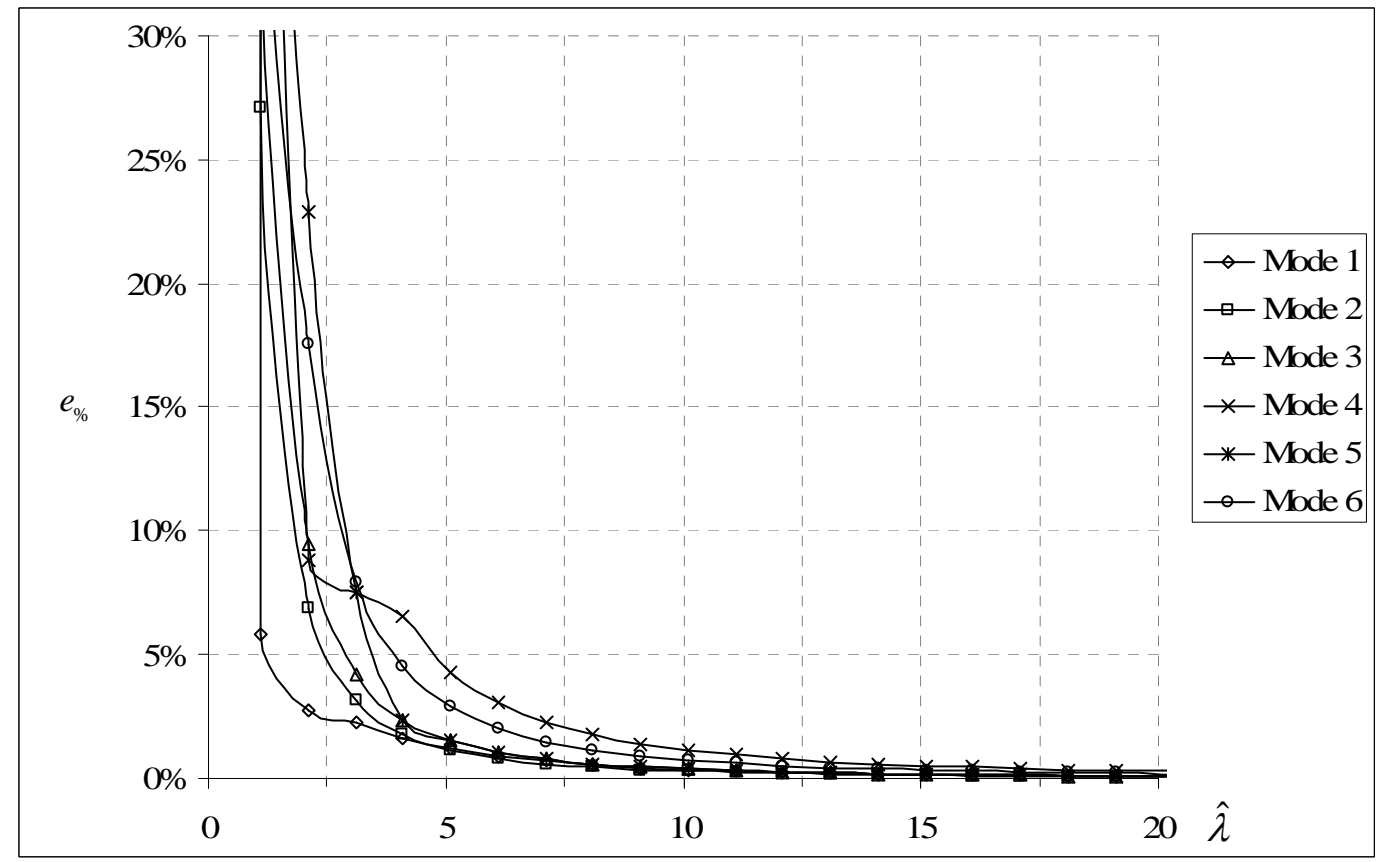

Figure 11. Cosserat versus Lattice: Relative error $e_{\%}$ of the various oscillation modes for wave propagating at $90^{\circ}\left(\hat{k}_{1}=0, \quad \hat{k}_{2}=\hat{k}\right) \cdot \hat{\lambda}$ is the wave length normalized by the block dimension $\alpha$.

Notice that for wavelengths seven times bigger the size $a$ of a block the error is less than $10 \%$. Consequently, we can say that the dynamic behavior of the obtained Cosserat model approximates well the one of the lattice model.

\section{Conclusions}

When dealing with blocky or layered structures or more generally with any structure where heterogeneities are present, one can address the question of modeling the behavior of such a structure either by considering each heterogeneity individually and solving the problem as in the Discrete Element Methods, or by considering the salient features of the discontinuum within the framework of generalized continuum theory. There are several techniques to formulate a continuum model based on the micromechanics of the structure. Here, we made use of the homogenization by differential expansions technique. The construction of the equivalent Cosserat continuum is based on the identification of the elastic energy stored in the lattice elementary cell with the one stored in the continuum. In that sense, the approach differs from the one of Sulem \& Mühlhaus 
[4], where the identification is performed directly on the dynamic equations. In the latter case, as also mentioned by Mühlhaus \& Oka [24], the higher order terms may introduce destabilizing negative terms in the deformation energy of the obtained continuum. This drawback is avoided in the present approach. Special attention has also been paid to the order of expansion of the translational field, which have to be one order higher than of the rotational field.

The obtained three-dimensional Cosserat continuum is validated by juxtaposing the dispersion functions of the lattice and the continuous model. In the lattice structure the block displacements and rotations are energy carriers in wave propagation. This is also the case in a Cosserat continuum, as opposed to the classical continuum, where only translational waves are considered. It is shown that the Cosserat continuum approximates well the lattice structure for wavelengths bigger than seven times the block size. However, the Cosserat model becomes increasingly inaccurate for smaller wavelengths. Generally one could assert that the Cosserat theory appears to be the natural starting point for the development of continuum models for blocky structures.

The derived constitutive law can be introduced into a finite element code in order to analyse real and complex structures. This application will be presented in a future publication. Three-dimensional multi-yield plasticity criteria will also be formulated in order to account for the limit strength of the building blocks and of the mortar and to describe the inter-block sliding, tilting and twisting failure modes of the brickwork. 


\section{APPENDIX}

\section{Lattice equations of motion}

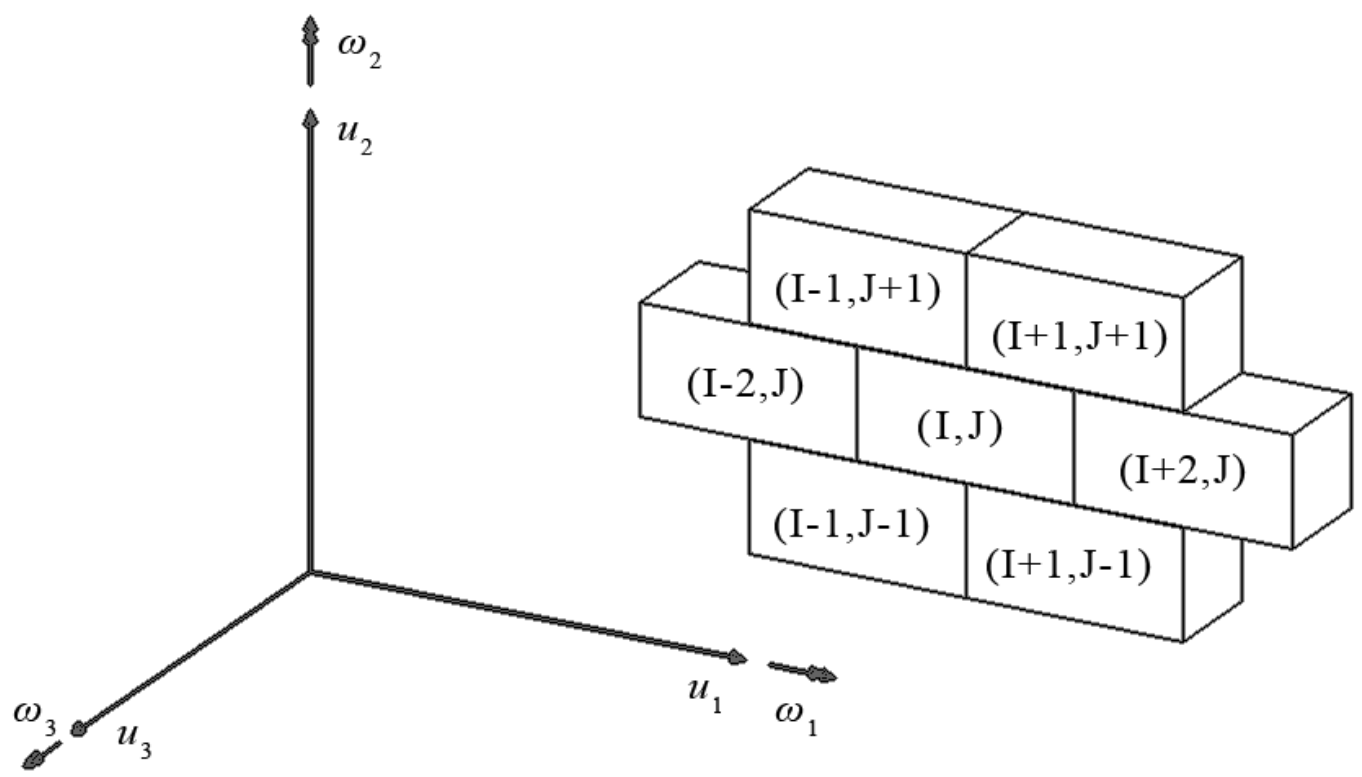

Figure 12. Geometrical configuration.

The equilibrium of forces and moments acting on block (I,J) yields to the following six equations:

$$
\begin{aligned}
m\left(\ddot{u}_{1}\right)_{\mathrm{I}, \mathrm{J}}^{b} & =A_{V} c_{N}\left(\left(u_{1}\right)_{\mathrm{I}-2, \mathrm{~J}}^{b}-2\left(u_{1}\right)_{\mathrm{I}, \mathrm{J}}^{b}+\left(u_{1}\right)_{\mathrm{I}+2, \mathrm{~J}}^{b}\right)+ \\
& +A_{H} c_{Q}\left(\left(u_{1}\right)_{\mathrm{I}-1, \mathrm{~J}-1}^{b}+\left(u_{1}\right)_{\mathrm{I}-1, \mathrm{~J}+1}^{b}-4\left(u_{1}\right)_{\mathrm{I}, \mathrm{J}}^{b}+\left(u_{1}\right)_{\mathrm{I}+1, \mathrm{~J}-1}^{b}+\left(u_{1}\right)_{\mathrm{I}+1, \mathrm{~J}+1}^{b}\right)+ \\
& +\frac{1}{2} A_{H} c_{Q} b\left(-\left(\omega_{3}\right)_{\mathrm{I}-1, \mathrm{~J}-1}^{b}+\left(\omega_{3}\right)_{\mathrm{I}-1, \mathrm{~J}+1}^{b}-\left(\omega_{3}\right)_{\mathrm{I}+1, \mathrm{~J}-1}^{b}+\left(\omega_{3}\right)_{\mathrm{I}+1, \mathrm{~J}+1}^{b}\right) \\
m\left(\ddot{u}_{2}\right)_{\mathrm{I}, \mathrm{J}}^{b}= & A_{H} c_{N}\left(\left(u_{2}\right)_{\mathrm{I}-1, \mathrm{~J}-1}^{b}+\left(u_{2}\right)_{\mathrm{I}-1, \mathrm{~J}+1}^{b}-4\left(u_{2}\right)_{\mathrm{I}, \mathrm{J}}^{b}+\left(u_{2}\right)_{\mathrm{I}+1, \mathrm{~J}-1}^{b}+\left(u_{2}\right)_{\mathrm{I}+1, \mathrm{~J}+1}^{b}\right)+ \\
+ & A_{V} c_{Q}\left(\left(u_{2}\right)_{\mathrm{I}-2, \mathrm{~J}}^{b}-2\left(u_{2}\right)_{\mathrm{I}, \mathrm{J}}^{b}+\left(u_{2}\right)_{\mathrm{I}+2, \mathrm{~J}}^{b}\right)+\frac{1}{2} A_{V} c_{Q} a\left(\left(\omega_{3}\right)_{\mathrm{I}-2, \mathrm{~J}}^{b}-\left(\omega_{3}\right)_{\mathrm{I}+2, \mathrm{~J}}^{b}\right)+ \\
+ & \frac{1}{4} A_{H} c_{N} a\left(\left(\omega_{3}\right)_{\mathrm{I}-1, \mathrm{~J}-1}^{b}+\left(\omega_{3}\right)_{\mathrm{I}-1, \mathrm{~J}+1}^{b}-\left(\omega_{3}\right)_{\mathrm{I}+1, \mathrm{~J}-1}^{b}-\left(\omega_{3}\right)_{\mathrm{I}+1, \mathrm{~J}+1}^{b}\right)
\end{aligned}
$$




$$
\begin{aligned}
m\left(\ddot{u}_{3}\right)_{\mathrm{I}, \mathrm{J}}^{b} & =A_{H} c_{Q}\left(\left(u_{3}\right)_{\mathrm{I}-1, \mathrm{~J}-1}^{b}+\left(u_{3}\right)_{\mathrm{I}-1, \mathrm{~J}+1}^{b}-4\left(u_{3}\right)_{\mathrm{I}, \mathrm{J}}^{b}+\left(u_{3}\right)_{\mathrm{I}+1, \mathrm{~J}-1}^{b}+\left(u_{3}\right)_{\mathrm{I}+1, \mathrm{~J}+1}^{b}\right)+ \\
& +A_{V} c_{Q}\left(\left(u_{3}\right)_{\mathrm{I}-2, \mathrm{~J}}^{b}-2\left(u_{3}\right)_{\mathrm{I}, \mathrm{J}}^{b}+\left(u_{3}\right)_{\mathrm{I}+2, \mathrm{~J}}^{b}\right)+ \\
& +\frac{1}{2} A_{H} c_{Q} b\left(\left(\omega_{1}\right)_{\mathrm{I}-1, \mathrm{~J}-1}^{b}-\left(\omega_{1}\right)_{\mathrm{I}-1, \mathrm{~J}+1}^{b}+\left(\omega_{1}\right)_{\mathrm{I}+1, \mathrm{~J}-1}^{b}-\left(\omega_{1}\right)_{\mathrm{I}+1, \mathrm{~J}+1}^{b}\right)+ \\
& +\frac{1}{4} A_{H} c_{Q} a\left(-\left(\omega_{2}\right)_{\mathrm{I}-1, \mathrm{~J}-1}^{b}-\left(\omega_{2}\right)_{\mathrm{I}-1, \mathrm{~J}+1}^{b}+\left(\omega_{2}\right)_{\mathrm{I}+1, \mathrm{~J}-1}^{b}+\left(\omega_{2}\right)_{\mathrm{I}+1, \mathrm{~J}+1}^{b}\right)+ \\
& +\frac{1}{2} A_{V} c_{Q} a\left(-\left(\omega_{2}\right)_{\mathrm{I}-2, \mathrm{~J}}^{b}+\left(\omega_{2}\right)_{\mathrm{I}+2, \mathrm{~J}}^{b}\right)
\end{aligned}
$$

$$
\begin{aligned}
& J_{1}\left(\ddot{\omega}_{1}\right)_{\mathrm{I}, \mathrm{J}}^{b}=A_{V} c_{\mathrm{MV} 1}\left(\left(\omega_{1}\right)_{\mathrm{I}-2, \mathrm{~J}}^{b}-2\left(\omega_{1}\right)_{\mathrm{I}, \mathrm{J}}^{b}+\left(\omega_{1}\right)_{\mathrm{I}+2, \mathrm{~J}}^{b}\right)+ \\
&+ A_{H} c_{\mathrm{MH} 1}\left(\left(\omega_{1}\right)_{\mathrm{I}-1, \mathrm{~J}-1}^{b}+\left(\omega_{1}\right)_{\mathrm{I}-1, \mathrm{~J}+1}^{b}-4\left(\omega_{1}\right)_{\mathrm{I}, \mathrm{J}}^{b}+\left(\omega_{1}\right)_{\mathrm{I}+1, \mathrm{~J}-1}^{b}+\left(\omega_{1}\right)_{\mathrm{I}+1, \mathrm{~J}+1}^{b}\right)- \\
&-\frac{1}{4} A_{H} c_{Q} b^{2}\left(+\left(\omega_{1}\right)_{\mathrm{I}-1, \mathrm{~J}-1}^{b}+\left(\omega_{1}\right)_{\mathrm{I}-1, \mathrm{~J}+1}^{b}+4\left(\omega_{1}\right)_{\mathrm{I}, \mathrm{J}}^{b}+\left(\omega_{1}\right)_{\mathrm{I}+1, \mathrm{~J}-1}^{b}+\left(\omega_{1}\right)_{\mathrm{I}+1, \mathrm{~J}+1}^{b}\right)- \\
&-\frac{1}{8} A_{H} c_{Q} b a\left(-\left(\omega_{2}\right)_{\mathrm{I}-1, \mathrm{~J}-1}^{b}+\left(\omega_{2}\right)_{\mathrm{I}-1, \mathrm{~J}+1}^{b}+\left(\omega_{2}\right)_{\mathrm{I}+1, \mathrm{~J}-1}^{b}-\left(\omega_{2}\right)_{\mathrm{I}+1, \mathrm{~J}+1}^{b}\right)- \\
&-\frac{1}{2} A_{H} c_{Q} b\left(\left(u_{3}\right)_{\mathrm{I}-1, \mathrm{~J}-1}^{b}-\left(u_{3}\right)_{\mathrm{I}-1, \mathrm{~J}+1}^{b}+\left(u_{3}\right)_{\mathrm{I}+1, \mathrm{~J}-1}^{b}-\left(u_{3}\right)_{\mathrm{I}+1, \mathrm{~J}+1}^{b}\right) \\
& J_{2}\left(\ddot{\omega}_{2}\right)_{\mathrm{I}, \mathrm{J}}^{b}=A_{V} c_{\mathrm{MV} 2}\left(\left(\omega_{2}\right)_{\mathrm{I}-2, \mathrm{~J}}^{b}-2\left(\omega_{2}\right)_{\mathrm{I}, \mathrm{J}}^{b}+\left(\omega_{2}\right)_{\mathrm{I}+2, \mathrm{~J}}^{b}\right)+ \\
&+ A_{H} c_{\mathrm{MH} 2}\left(\left(\omega_{2}\right)_{\mathrm{I}-1, \mathrm{~J}-1}^{b}+\left(\omega_{2}\right)_{\mathrm{I}-1, \mathrm{~J}+1}^{b}-4\left(\omega_{2}\right)_{\mathrm{I}, \mathrm{J}}^{b}+\left(\omega_{2}\right)_{\mathrm{I}+1, \mathrm{~J}-1}^{b}+\left(\omega_{2}\right)_{\mathrm{I}+1, \mathrm{~J}+1}^{b}\right)- \\
&- \frac{1}{4} A_{V} c_{Q} a^{2}\left(\left(\omega_{2}\right)_{\mathrm{I}-2, \mathrm{~J}}^{b}+2\left(\omega_{2}\right)_{\mathrm{I}, \mathrm{J}}^{b}+\left(\omega_{2}\right)_{\mathrm{I}+2, \mathrm{~J}}^{b}\right)- \\
&- \frac{1}{16} A_{H} c_{Q} a^{2}\left(+\left(\omega_{2}\right)_{\mathrm{I}-1, \mathrm{~J}-1}^{b}+\left(\omega_{2}\right)_{\mathrm{I}-1, \mathrm{~J}+1}^{b}+4\left(\omega_{2}\right)_{\mathrm{I}, \mathrm{J}}^{b}+\left(\omega_{2}\right)_{\mathrm{I}+1, \mathrm{~J}-1}^{b}+\left(\omega_{2}\right)_{\mathrm{I}+1, \mathrm{~J}+1}^{b}\right)- \\
&- \frac{1}{8} A_{H} c_{Q} a b\left(-\left(\omega_{1}\right)_{\mathrm{I}-1, \mathrm{~J}-1}^{b}+\left(\omega_{1}\right)_{\mathrm{I}-1, \mathrm{~J}+1}^{b}+\left(\omega_{1}\right)_{\mathrm{I}+1, \mathrm{~J}-1}^{b}-\left(\omega_{1}\right)_{\mathrm{I}+1, \mathrm{~J}+1}^{b}\right)- \\
&- \frac{1}{2} A_{V} c_{Q} a\left(-\left(u_{3}\right)_{\mathrm{I}-2, \mathrm{~J}}^{b}+\left(u_{3}\right)_{\mathrm{I}+2, \mathrm{~J}}^{b}\right)- \\
&- \frac{1}{4} A_{H} a c_{Q}\left(-\left(u_{3}\right)_{\mathrm{I}-1, \mathrm{~J}-1}^{b}-\left(u_{3}\right)_{\mathrm{I}-1, \mathrm{~J}+1}^{b}+\left(u_{3}\right)_{\mathrm{I}+1, \mathrm{~J}-1}^{b}+\left(u_{3}\right)_{\mathrm{I}+1, \mathrm{~J}+1}^{b}\right)
\end{aligned}
$$

29 


$$
\begin{aligned}
J_{3}\left(\ddot{\omega}_{3}\right)_{\mathrm{I}, \mathrm{J}}^{b}=A_{V} c_{\mathrm{MV} 3}\left(\left(\omega_{3}\right)_{\mathrm{I}-2, \mathrm{~J}}^{b}-2\left(\omega_{3}\right)_{\mathrm{I}, \mathrm{J}}^{b}+\left(\omega_{3}\right)_{\mathrm{I}+2, \mathrm{~J}}^{b}\right)+ \\
+c_{\mathrm{MH} 3} A_{H}\left(\left(\omega_{3}\right)_{\mathrm{I}-1, \mathrm{~J}-1}^{b}+\left(\omega_{3}\right)_{\mathrm{I}-1, \mathrm{~J}+1}^{b}-4\left(\omega_{3}\right)_{\mathrm{I}, \mathrm{J}}^{b}+\left(\omega_{3}\right)_{\mathrm{I}+1, \mathrm{~J}-1}^{b}+\left(\omega_{3}\right)_{\mathrm{I}+1, \mathrm{~J}+1}^{b}\right)- \\
-\frac{1}{16} A_{H} c_{N} a^{2}\left(\left(\omega_{3}\right)_{\mathrm{I}-1, \mathrm{~J}-1}^{b}+\left(\omega_{3}\right)_{\mathrm{I}-1, \mathrm{~J}+1}^{b}+4\left(\omega_{3}\right)_{\mathrm{I}, \mathrm{J}}^{b}+\left(\omega_{3}\right)_{\mathrm{I}+1, \mathrm{~J}-1}^{b}+\left(\omega_{3}\right)_{\mathrm{I}+1, \mathrm{~J}+1}^{b}\right)- \\
-\frac{1}{4} A_{H} c_{Q} b^{2}\left(\left(\omega_{3}\right)_{\mathrm{I}-1, \mathrm{~J}-1}^{b}+\left(\omega_{3}\right)_{\mathrm{I}-1, \mathrm{~J}+1}^{b}+4\left(\omega_{3}\right)_{\mathrm{I}, \mathrm{J}}^{b}+b\left(\omega_{3}\right)_{\mathrm{I}+1, \mathrm{~J}-1}^{b}+\left(\omega_{3}\right)_{\mathrm{I}+1, \mathrm{~J}+1}^{b}\right)- \\
-\frac{1}{4} A_{V} c_{Q} a^{2}\left(\left(\omega_{3}\right)_{\mathrm{I}-2, \mathrm{~J}}^{b}+2\left(\omega_{3}\right)_{\mathrm{I}, \mathrm{J}}^{b}+\left(\omega_{3}\right)_{\mathrm{I}+2, \mathrm{~J}}^{b}\right)- \\
-\frac{1}{4} A_{H} c_{N} a\left(\left(u_{2}\right)_{\mathrm{I}-1, \mathrm{~J}-1}^{b}+\left(u_{2}\right)_{\mathrm{I}-1, \mathrm{~J}+1}^{b}-\left(u_{2}\right)_{\mathrm{I}+1, \mathrm{~J}-1}^{b}-\left(u_{2}\right)_{\mathrm{I}+1, \mathrm{~J}+1}^{b}\right)- \\
-\frac{1}{2} A_{H} c_{Q} b\left(-\left(u_{1}\right)_{\mathrm{I}-1, \mathrm{~J}-1}^{b}+\left(u_{1}\right)_{\mathrm{I}-1, \mathrm{~J}+1}^{b}-\left(u_{1}\right)_{\mathrm{I}+1, \mathrm{~J}-1}^{b}+\left(u_{1}\right)_{\mathrm{I}+1, \mathrm{~J}+1}^{b}\right)- \\
-\frac{1}{2} A_{V} c_{Q} a\left(\left(u_{2}\right)_{\mathrm{I}-2, \mathrm{~J}}^{b}-\left(u_{2}\right)_{\mathrm{I}+2, \mathrm{~J}}^{b}\right)
\end{aligned}
$$

\section{Acknowledgments}

This research is sponsored by the General Secretariat for Research and Technology in Greece and the French Ministry of Foreign Affairs in the frame of the bilateral S \& T cooperation between the French and Hellenic Republic (2005-2007): "Nouvelles méthodes d'analyse numérique du comportement mécanique des monuments anciens - Application à l'Acropole". The authors would like to thank K. Sab for fruitful discussions and the two reviewers for their constructive remarks and suggestions.

\section{References}

[1] D. Besdo, Inelastic behaviour of plane frictionless block-systems described as Cosserat media, Arch. Mech, Meccanica 37 (1985), pp. 603-619

[2] R. Masiani, N.L. Rizzi and P.Trovalusci, Masonry walls as structured continua, Meccanica 30 (1995), pp. 673-683

[3] R. Masiani and P.Trovalusci, Cosserat and Cauchy materials as continuum models of brick masonry, Meccanica 31 (1996), pp. 421-432

[4] J. Sulem and H.-B. Mühlhaus, A continuum model for periodic two-dimensional block structures, Mechanics of Cohesive-Frictional Materials, 2 (1997) pp. 31-46 
[5] F. Pradel and K. Sab, Cosserat modeling of elastic periodic lattice structures, C. R. Acad. Sci. Paris, Série II b, 326 (1998), pp. 699-704

[6] A. Cecchi and K.Sab, A multi-parameter homogenization study for modeling elastic masonry, European Journal of Mechanics 21 (2002), pp. 249-268

[7] A. Cecchi and K.Sab, Out of plane model for heterogeneous periodic materials: the case of masonry, European Journal of Mechanics 21 (2002), pp. 715-746

[8] A. Cecchi and K.Sab, A comparison between a 3D discrete model and two homogenized plate models for periodic elastic brickwork, International Journal of Solids and Structures 41 (2004), pp. 2259-2276

[9] P. Trovalusci and R. Masiani, A multifield model for blocky materials based on multiscale description, International Journal of Solids and Structures 42 (2005), pp. 5778-5794

[10] A. Bensoussan, J.L. Lions and G. Papanicolaou, Asymptotic Analysis for Periodic Structures, North-Holland Publ. Co., Amsterdam, 1978

[11] E. Sanchez-Palencia, Non-homogeneous Media and Vibration Theory, Springer-Verlag, Berlin, 1980

[12] E. Sanchez-Palencia and A. Zaoui, Homogenization Techniques for Composite Media, Springer-Verlag, Berlin, 1987

[13] N. Bakhalov and G. Panasenko, Homogenisation: Averaging Process in Periodic Media: Mathematical Problems in the Mechanics of Composite Materials, Kluwer Academic Publishers, Dordrecht-Boston-London, 1989

[14] A.L. Kalamkarov, Composite and Reinforced Elements of Construction, Wiley, New York, 1992

[15] H. Tollenaere and D. Caillerie, Continuous modeling of lattice structures by homogenization, Advances in Engineering Software, 29:7-9 (1998) pp. 699-705

[16] Z. Hashin, The Elastic Moduli of Heterogeneous Materials, ASME Journal of Applied Mechanics, 29 (1962) pp. 143-150

[17] Z. Hashin and S. Shtrikman, A Variational Approach to the Theory of the Elastic Behaviours of Multiphase Materials, Journal of the Mechanics and Physics of Solids, 11 (1963) pp. 127-140

[18] R. Hill, Elastic Properties of Reinforced Solids. Some Theoretical Principles, Journal of the Mechanics and Physics of Solids, 11 (1963) pp. 357-372 
[19] R. Hill, A Self-consistent Mechanics of Composite Materials, Journal of the Mechanics and Physics of Solids, 13 (1965) pp. 213

[20] R.M. Christensen, Mechanics of Composite Materials, John Wiley, New York, 1979

[21] J. Aboudi, Mechanics of Composite Materials. A Unified Micromechanical Approach, Elsevier, Amsterdam-Oxford-New York-Tokyo, 1991

[22] I.A. Kunin, Elastic Media with Microstructure I. One-dimensional Models, Berlin, Heidelberg, New York, Springer-Verlag, 1982

[23] I.A. Kunin, Elastic Media with Microstructure II. Three-dimensional Models, Berlin, Heidelberg, New York, Springer-Verlag, 1983

[24] H.B. Mühlhaus and F. Oka, Dispersion and wave propagation in discrete and continuous models for granular materials, International Journal of Solids and Structures, 33 (1996) pp. 28412858

[25] G.A. Maugin, Non-Linear Waves in Elastic Crystals, Oxford: Oxford University Press,1999

[26] A.S.J. Suiker, R. de Borst and C.S. Chang, Micro-mechanically based higher-order continuum models for granular materials, In: D. Kolymbas (ed.), Constitutive Modelling of Granular Materials. Berlin: Springer (2000) pp. 249-274

[27] A.S.J. Suiker, R. de Borst and C.S. Chang, Micro-mechanical modelling of granular material. Part 1: Derivation of a second-gradient micro-polar constitutive theory, Acta Mechanica 149 (2001) pp. 161-180

[28] A.S.J. Suiker, R. de Borst and C.S. Chang, Micro-mechanical modelling of granular material. Part 2: Plane wave propagation in finite media, Acta Mechanica 149 (2001) pp. 181-200

[29] E. Pasternak and H.-B. Mühlhaus, Generalised homogenization procedures for granular material, Journal of Engineering Mathematics 52 (2005) pp. 199-229

[30] H.B. Mühlhaus, Continuum models for layered and blocky rock, In: C. Fairhurst (ed.), Comprehensive Rock Engineering, 2 (1993) pp. 209-230

[31] H.B. Mühlhaus, J. Sulem and P. Unterreiner, Discrete and continuous models for dry masonry columns, ASCE Journal of Engineering Mechanics, 123 (1997) pp. 399-403

[32] M. Cerrolaza, J. Sulem and A. Elbied, A Cosserat non-linear finite element analysis software for blocky structure, Advances in Engineering Software, 30 (1999) pp. 69-83 
[33] I. Stefanou, J. Sulem and I.Vardoulakis, Continuum modelling of masonry structures under static and dynamic loading, In: S.K. Kourkoulis (ed.), Fracture and Failure of Natural Building Stones, Springer, Doordrecht, the Netherlands (2006) pp. 123-136

[34] D.P. Adhikary, H.B. Mühlhaus and A.V. Dyskin, A numerical study of flexural buckling of foliated rock slopes, International Journal for Numerical and Analytical Methods in Geomechanics 25 (2001) pp. 871-884

[35] I. Vardoulakis and J. Sulem, Bifurcation Analysis in Geomechanics, Blackie Academic and Professional, Glascow, 1995

[36] Wolfram Research, Inc, Mathematica ${ }^{\circledR}$ 5.0, (C) Copyright 1988-2005

[37] D. Raffard, Modélisation de structures maçonnées par homogénéisation numérique non linéaire: application aux ouvrages d'intérêt archéologique, $\mathrm{PhD}$ Thesis at Institut National Polytechnique de Lorraine, France (2000)

[38] G. Milani, P.B. Lourenco and A. Tralli, Homogenised limit analysis of masonry walls, Part I: Failure surfaces, Computers and Structures 84 (2006) pp. 166-180

[39] V.V. Novozhilov, Theory of Elasticity, Pergamon Press, 1961

[40] A. Askar and A.S. Cakmak, A structural model of a micropolar continuum, International Journal of Engineering Science 6 (1968) pp. 583-589

[41] H.J. Pain, The Physics of Vibrations and Waves, Forth Edition, John Wiley \& Sons, New York, 1995 